\title{
Rigidity and Positivity of Mass for Asymptotically Hyperbolic Manifolds
}

\author{
Lars Andersson, Mingliang Cai, and Gregory J. Galloway
}

\begin{abstract}
The Witten spinorial argument has been adapted in several works over the years to prove positivity of mass in the asymptotically AdS and asymptotically hyperbolic settings in arbitrary dimensions. In this paper we prove a scalar curvature rigidity result and a positive mass theorem for asymptotically hyperbolic manifolds that do not require a spin assumption. The positive mass theorem is reduced to the rigidity case by a deformation construction near the conformal boundary. The proof of the rigidity result is based on a study of minimizers of the BPS brane action.
\end{abstract}

\section{Introduction}

Developments in string theory during the past decade, in particular the emergence of the AdS/CFT correspondence, have increased interest in the mathematical and physical properties of asymptotically hyperbolic Riemannian manifolds. Such manifolds arise naturally as spacelike hypersurfaces in asymptotically anti-de Sitter spacetimes.

Asymptotically hyperbolic manifolds have a rich geometry at infinity, as exhibited by, e.g., renormalized volume and $Q$-curvature. The mass of an asymptotically hyperbolic manifold may, under suitable asymptotic conditions, be defined as the integral of a function defined at infinity, the so-called mass aspect function. This feature is related to the fact that, in contrast to the asymptotically Euclidean case, bounded harmonic functions on an AH manifold are not in general constant, but have nontrivial boundary values at infinity.

In this paper we shall prove a scalar curvature rigidity result and a positive mass theorem for asymptotically hyperbolic manifolds. The results do not require a spin assumption. The positive mass theorem is reduced to the rigidity case by a novel deformation construction near the conformal boundary. The proof of the rigidity result is based on a study of minimizers of the BPS brane action. 
Let $\left(M^{n+1}, g\right)$ denote an $(n+1)$-dimensional Riemannian manifold, and let $\mathbb{H}^{n+1}$ denote $(n+1)$-dimensional hyperbolic space of curvature $K=-1$. As a prelude to proving positivity of mass in the asymptotically hyperbolic setting (see the discussion below), we first establish the following rigidity result.

Theorem 1.1. Suppose $\left(M^{n+1}, g\right), 2 \leq n \leq 6$, has scalar curvature $S[g]$ satisfying, $S[g] \geq-n(n+1)$, and is isometric to $\mathbb{H}^{n+1}$ outside a compact set. Then $\left(M^{n+1}, g\right)$ is globally isometric to $\mathbb{H}^{n+1}$.

In the case that $\left(M^{n+1}, g\right)$ is a spin manifold, this theorem follows from a result of Min-oo [19] (see also [2,13]), as well as from the rigidity part of the more recently proved positive mass theorem for asymptotically hyperbolic manifolds $[10$, 26]. The main point of Theorem 1.1 is that it does not require a spin assumption. We note, for comparison, that there have been some other recently obtained rigidity results for hyperbolic space $[4,7,21,24]$ that do not require a spin assumption, but these impose conditions on the Ricci curvature.

The proof of Theorem 1.1 is based on the general minimal surface methodology of Schoen and Yau [22], adapted to a negative lower bound on the scalar curvature. This means, in our approach, that minimal surfaces are replaced by non-zero constant mean curvature surfaces, and the area functional is replaced by the so-called BPS brane action, as utilized by Witten and Yau [27] in their work on the AdS/CFT correspondence. From the regularity results of geometric measure theory, we require $M$ to have dimension $\leq 7$ in order to avoid the occurrence of singularities in co-dimension one minimizers of the brane action ${ }^{1}$. In Section 2 we prove a local warped product splitting result, where the splitting takes place about a certain minimizer of the brane action. This splitting result, which extends to the case of negative lower bound on the scalar curvature previous results of Cai and Galloway [5,6], is then used to prove Theorem 1.1.

Our original motivation for proving Theorem 1.1 was to obtain a proof of positivity of mass for asymptotically hyperbolic manifolds that does not require a spin assumption. In [15], Gibbons et al. adapted Witten's spinorial argument to prove positivity of mass in the $3+1$ asymptotically AdS setting. More recently, Wang [26], and, under weaker asymptotic conditions, Chruściel and Herzlich [10] have provided precise definitions of the mass in the asymptotically hyperbolic setting and have given spinor based proofs of positivity of mass in dimensions $\geq 3$. These latter positive mass results may be paraphrased as follows:

Theorem 1.2. Suppose $\left(M^{n+1}, g\right), n \geq 2$, is an asymptotically hyperbolic spin manifold with scalar curvature $S \geq-n(n+1)$. Then $M$ has mass $m \geq 0$, and $=0$ iff $M$ is isometric to standard hyperbolic space $\mathbb{H}^{n+1}$.

\footnotetext{
${ }^{1}$ However, the work of Christ and Lohkamp $[8,17]$ offers the possibility of eliminating this di-
} mension restriction. 
Physically, $M$ corresponds to a maximal (mean curvature zero) spacelike hypersurface in spacetime satisfying the Einstein equations with cosmological constant $\Lambda=-n(n+1) / 2$. For then the Gauss equation and weak energy condition imply $S \geq-n(n+1)$.

Here we present the following version of Theorem 1.2, which does not require $M$ to be spin.

Theorem 1.3. Let $\left(M^{n+1}, g\right), 2 \leq n \leq 6$, be an asymptotically hyperbolic manifold with scalar curvature $S[g] \geq-n(n+1)$. Assume that the mass aspect function does not change sign, i.e., that it is either negative, zero, or positive. Then, either the mass of $(M, g)$ is positive, or $(M, g)$ is isometric to hyperbolic space.

As noted above, the mass aspect function is a scalar function whose integral over conformal infinity determines the mass; see Section 3 for precise definitions.

Our approach to proving Theorem 1.3 is inspired by Lohkamp's variation [18] of the Schoen-Yau [23] proof of the classical positive mass theorem for asymptotically flat manifolds. Our proof makes use of Theorem 1.1, together with a deformation result, which shows roughly that if an asymptotically hyperbolic manifold with scalar curvature satisfying, $S \geq-n(n+1)$, has negative mass aspect then the metric can be deformed near infinity to the hyperbolic metric, while maintaining the scalar curvature inequality. This deformation result (Theorem 3.2), along with an analysis of the case in which the mass aspect vanishes identically (Theorem 3.9), and their application to the proof of Theorem 1.3 are presented in Section 3.

\section{The rigidity result}

The aim of this section is to give a proof of Theorem 1.1.

\subsection{The brane action}

Let $\left(M^{n+1}, g\right)$ be an $(n+1)$-dimensional oriented Riemannian manifold with volume form $\Omega$. Assume there is a globally defined form $\Lambda$ such that $\Omega=d \Lambda$.

Let $\Sigma^{n}$ be a compact orientable hypersurface in $M$. Then $\Sigma$ is 2-sided in $M$; designate one side as the "outside" and the other as the "inside". Let $\nu$ be the outward pointing unit normal along $\Sigma$, and let $\Sigma$ have the orientation induced by $\nu$ (i.e., determined by the induced volume form $\omega=i_{\nu} \Omega$ ). Then, for any such $\Sigma$, we define the brane action $\mathcal{B}$ by,

$$
\mathcal{B}(\Sigma)=\mathcal{A}(\Sigma)-n \mathcal{V}(\Sigma),
$$

where $\mathcal{A}(\Sigma)=$ the area of $\Sigma$, and $\mathcal{V}(\Sigma)=\int_{\Sigma} \Lambda$. If $\Sigma$ bounds to the inside then, by Stokes theorem, $\mathcal{V}(\Sigma)=$ the volume of the region enclosed by $\Sigma$. Although $\Lambda$ is not uniquely determined, Stokes theorem shows that, within a given homology class, $\mathcal{B}$ is uniquely determined up to an additive constant.

We wish to consider the formulas for the first and second variation of the brane action. First, to fix notations, let $A$ denote the second fundamental form 
of $\Sigma$; by our conventions, for each pair of tangent vectors $X, Y \in T_{p} \Sigma$,

$$
A(X, Y)=\left\langle\nabla_{X} \nu, Y\right\rangle,
$$

where $\nabla$ is the Levi-Civita connection of $(M, g)$ and $h=\langle$,$\rangle is the induced metric$ on $\Sigma$. Then $H=\operatorname{tr} A$ is the mean curvature of $\Sigma$.

Let $t \rightarrow \Sigma_{t},-\epsilon<t<\epsilon$, be a normal variation of $\Sigma=\Sigma_{0}$, with variation vector field $V=\left.\frac{\partial}{\partial t}\right|_{t=0}=\phi \nu, \phi \in C^{\infty}(\Sigma)$. Abusing notation slightly, set $\mathcal{B}(t)=$ $\mathcal{B}\left(\Sigma_{t}\right)$. Then for first variation we have,

$$
\mathcal{B}^{\prime}(0)=\int_{\Sigma}(H-n) \phi d A .
$$

Thus $\Sigma$ is a stationary point for the brane action if and only if it has constant mean curvature $H=n$.

Assuming $\Sigma$ has mean curvature $H=n$, the second variation formula is given by

$$
\mathcal{B}^{\prime \prime}(0)=\int_{\Sigma} \phi L(\phi) d A
$$

where,

$$
L(\phi)=-\triangle \phi+\frac{1}{2}\left(S_{\Sigma}-S-|A|^{2}-H^{2}\right) \phi,
$$

and where $S_{\Sigma}$ is the scalar curvature of $\Sigma$ and $S$ is the scalar curvature of $M$. Here $L$ is the stability operator associated with the brane action, and is closely related to the stability operator of minimal surface theory. Using the fact that $H=n, L$ can be re-expressed as,

$$
L(\phi)=-\triangle \phi+\frac{1}{2}\left(S_{\Sigma}-S_{n}-\left|A_{0}\right|^{2}\right) \phi,
$$

where $S_{n}=S+n(n+1)$ and $A_{0}$ is the trace free part of $A, A_{0}=A-h$. We note that, in our applications, $S_{n}$ will be nonnegative.

A stationary point $\Sigma$ for the brane action is said to be $\mathcal{B}$-stable provided for all normal variations $t \rightarrow \Sigma_{t}$ of $\Sigma, \mathcal{B}^{\prime \prime}(0) \geq 0$. For operators of the form (2.6), the following proposition is well-known.

Proposition 2.1. The following conditions are equivalent.

1. $\Sigma$ is $\mathcal{B}$-stable.

2. $\lambda_{1} \geq 0$, where $\lambda_{1}$ is the principal eigenvalue of $L$.

3. There exists $\phi \in C^{\infty}(\Sigma), \phi>0$, such that $L(\phi) \geq 0$.

In particular, if $\lambda_{1} \geq 0, \phi$ in part 3 can be chosen to be an eigenfunction.

\subsection{Warped product splitting}

In this section we prove the local warped product splitting result alluded to in the introduction. As a precursor, we prove the following infinitesimal rigidity result. 
Proposition 2.2. Let $\left(M^{n+1}, g\right)$ be an oriented Riemannian manifold with scalar curvature $S$ satisfying,

$$
S \geq-n(n+1) .
$$

Let $\Sigma^{n}$ be a compact orientable $\mathcal{B}$-stable hypersurface in $M$ which does not admit a metric of positive scalar curvature. Then the following must hold.

(i) $\Sigma$ is umbilic, in fact $A=h$, where $h$ is the induced metric on $\Sigma$.

(ii) $\Sigma$ is Ricci flat and $S=-n(n+1)$ along $\Sigma$.

Proof. By Proposition 2.1, there exists $\phi \in \Sigma, \phi>0$, such that $L(\phi) \geq 0$. The scalar curvature $\tilde{S}$ of $\Sigma$ in the conformally rescaled metric $\tilde{h}=\phi^{\frac{2}{n-2}} h$ is then given by,

$$
\begin{aligned}
\tilde{S} & =\phi^{-\frac{n}{n-2}}\left(-2 \triangle \phi+S_{\Sigma} \phi+\frac{n-1}{n-2} \frac{|\nabla \phi|^{2}}{\phi}\right) \\
& =\phi^{-\frac{2}{n-2}}\left(2 \phi^{-1} L(\phi)+S_{n}+\left|A_{0}\right|^{2}+\frac{n-1}{n-2} \frac{|\nabla \phi|^{2}}{\phi^{2}}\right)
\end{aligned}
$$

where, for the second equation, we have used (2.6) with $f=\phi$. Since all terms in parentheses above are nonnegative, (2.8) implies that $\tilde{S} \geq 0$. If $\tilde{S}>0$ at some point, then by well known results [16] one can conformally change $\tilde{h}$ to a metric of strictly positive scalar curvature, contrary to assumption. Thus $\tilde{S}$ vanishes identically, which implies $L(\phi)=0, S_{n}=0, A_{0}=0$ and $\phi$ is constant. Equation (2.6), with $f=\phi$ then implies that $S \equiv 0$. By a result of Bourguignon (see [16]), it follows that $\Sigma$ carries a metric of positive scalar curvature unless it is Ricci flat. Thus conditions (i) and (ii) are satisfied.

Proposition 1.1 will be used in the proof of the following local warped product splitting result.

Theorem 2.3. Let $\left(M^{n+1}, g\right)$ be an oriented Riemannian manifold with scalar curvature $S \geq-n(n+1)$. Let $\Sigma$ be a compact orientable hypersurface in $M$ which does not admit a metric of positive scalar curvature. If $\Sigma$ locally minimizes the brane action $\mathcal{B}$ then there is a neighborhood $U$ of $\Sigma$ such that $\left(U,\left.g\right|_{U}\right)$ is isometric to the warped product $\left((-\epsilon, \epsilon) \times \Sigma, d t^{2}+e^{2 t} h\right)$, where $h$, the induced metric on $\Sigma$, is Ricci flat.

By "locally minimizes" we mean, for example, that $\Sigma$ has brane action less than or equal to that of all graphs over $\Sigma$ with respect to Gaussian normal coordinates. A related result has been obtained by Yau [28] in dimension three.

Proof. Let $\mathcal{H}(u)$ denote the mean curvature of the hypersurface $\Sigma_{u}: x \rightarrow \exp _{x} u(x) \nu$, $u \in C^{\infty}(\Sigma), u$ sufficiently small. $\mathcal{H}$ has linearization $\mathcal{H}^{\prime}(0)=L$, where $L$ is the $\mathcal{B}$-stability operator (2.6). But by Proposition 2.2, $L$ reduces to $-\triangle$, and hence $\mathcal{H}^{\prime}(0)=-\triangle$. We introduce the operator,

$$
\mathcal{H}^{*}: C^{\infty}(\Sigma) \times \mathbb{R} \rightarrow C^{\infty}(\Sigma) \times \mathbb{R}, \quad \mathcal{H}^{*}(u, k)=\left(\mathcal{H}(u)-k, \int_{\Sigma} u\right),
$$


which one easily checks has invertible linearization about $(0,0)$, since the kernel of $\mathcal{H}^{\prime}(0)$ contains only the constants. By the inverse function theorem, for each $\tau$ sufficiently small there exists $u=u_{\tau}$ and $k=k_{\tau}$ such that $\mathcal{H}\left(u_{\tau}\right)=k_{\tau}$ and $\int_{\Sigma} u_{\tau} d A=\tau$. Since $u^{\prime}(0) \in \operatorname{ker} \mathcal{H}^{\prime}(0)$, the latter equation implies that $u^{\prime}(0)=$ const $>0$. Thus for $\tau$ sufficiently small, the hypersurfaces $\Sigma_{u_{\tau}}$ form a foliation of a neighborhood $U$ of $\Sigma$ by constant mean curvature hypersurfaces.

Using coordinates on $\Sigma$ and the normal field to the $\Sigma_{u_{\tau}}$ 's to transport these coordinates to each $\Sigma_{u_{\tau}}$, we have, up to isometry,

$$
U=(-\epsilon, \epsilon) \times\left.\Sigma \quad g\right|_{U}=\phi^{2} d t^{2}+h_{t},
$$

where $h_{t}=h_{i j}(t, x) d x^{i} d x^{j}, \phi=\phi(t, x)$ and $\Sigma_{t}=\{t\} \times \Sigma$ has constant mean curvature. Since $\Sigma$ locally minimizes the brane action, we have, $\mathcal{B}(0) \leq \mathcal{B}(t)$ for all $t \in(-\epsilon, \epsilon)$, for $\epsilon$ sufficiently small.

Let $H(t)$ denote the mean curvature of $\Sigma_{t} . H=H(t)$ obeys the evolution equation,

$$
\frac{d H}{d t}=L(\phi),
$$

where for each $t, L$ is the operator on $\Sigma_{t}$ given in Equation (2.5). Since $\Sigma$ locally minimizes the brane action, we have $H(0)=n$. We show $H \leq n$ for $t \in[0, \epsilon)$. If this is not the case, there exists $t_{0} \in(0, \epsilon)$ such that $H\left(t_{0}\right)>n$. Moreover, $t_{0}$ can be chosen so that $H^{\prime}\left(t_{0}\right)>0$. Let $\tilde{S}$ be the scalar curvature of $\Sigma_{t_{0}}$ in the conformally related metric $\tilde{h}=\phi^{\frac{2}{n-2}} h_{t_{0}}$. Arguing similarly as in the derivation of (2.8), Equations (2.5) and (2.11) imply,

$$
\tilde{S}=\phi^{-\frac{2}{n-2}}\left(2 \phi^{-1} H^{\prime}\left(t_{0}\right)+S+|A|^{2}+H^{2}+\frac{n-1}{n-2} \frac{|\nabla \phi|^{2}}{\phi^{2}}\right),
$$

where all terms are evaluated on $\Sigma_{t_{0}}$. The Schwartz inequality gives, $|A|^{2} \geq$ $H^{2} / n>n$. This, together with the assumed scalar curvature inequality (2.7), implies that $S+|A|^{2}+H^{2}>0$. We conclude from (2.12) that $\Sigma_{t_{0}}$ carries a metric of positive scalar curvature, contrary to assumption.

Thus, $H \leq n$ on $[0, \epsilon)$, as claimed. Now, by the formula for the first variation of the brane action, it follows that

$$
\mathcal{B}^{\prime}(t)=\int_{\Sigma_{t}}(H-n) \phi d A \leq 0, \quad \text { for all } t \in[0, \epsilon) .
$$

But since $\mathcal{B}$ achieves a minimum at $t=0$, it must be that $\mathcal{B}^{\prime}(t)=0$ for $t \in[0, \epsilon)$. Hence, the integral in (2.13) vanishes, which implies that $H=n$ on $[0, \epsilon)$. A similar argument shows that $H=n$ on $(-\epsilon, 0]$, as well. Equation (2.11) then implies that $L(\phi)=0$ on each $\Sigma_{t}$. Hence, by Proposition 2.1 , each $\Sigma_{t}$ is $\mathcal{B}$-stable. From Proposition 2.2, we have that $A_{t}=h_{t}$, where $A_{t}$ is the second fundamental form of $\Sigma_{t}$, and that $\phi$ only depends on $t$. By a simple change of $t$-coordinate in (2.10), we may assume without loss of generality that $\phi=1$. Then the condition $A_{t}=h_{t}$ becomes, in the coordinates $(2.10), \frac{\partial h_{i j}}{\partial t}=2 h_{i j}$. Upon integration this gives, $h_{i j}(t, x)=e^{2 t} h_{i j}(0, x)$, which completes the proof of the theorem. 


\subsection{Proof of the rigidity result}

In order to prove Theorem 1.1 it is convenient to work with an explicit representation of hyperbolic space $\mathbb{H}^{n+1}$. We start with the half-space model $\left(H^{n+1}, g_{H}\right)$, where, $H^{n+1}=\left\{\left(y, x^{1}, \ldots, x^{n}\right): y>0\right\}$, and

$$
g_{H}=\frac{1}{y^{2}}\left(d y^{2}+\left(d x^{1}\right)^{2}+\cdots+\left(d x^{n}\right)^{2}\right),
$$

and make the change of variable $y=e^{-t}$, to obtain $\mathbb{H}^{n+1}=\left(\mathbb{R}^{n+1}, g_{0}\right)$, where,

$$
g_{0}=d t^{2}+e^{2 t}\left(\left(d x^{1}\right)^{2}+\cdots+\left(d x^{n}\right)^{2}\right) .
$$

As in the statement of Theorem 1.1 , let $\left(M^{n+1}, g\right)$ be a Riemannian manifold with scalar curvature $S[g]$ satisfying $S[g] \geq-n(n+1)$. We assume that there are compact sets $K \subset M, K_{0} \subset \mathbb{R}^{n+1}$ such that $M-K$ is diffeomorphic to $\mathbb{R}^{n+1}$ $K_{0}$, and, with respect to Cartesian coordinates $\left(t, x_{1}, \ldots, x_{n}\right)$ on the complement of $K, g=g_{0}$. We want to show that $\left(M^{n+1}, g\right)$ is globally isometric to $\mathbb{H}^{n+1}$. Since $M$ is simply connected near infinity, it is in fact sufficient to show that $M$ has everywhere constant curvature $K_{M}=-1$. Our approach to proving this is to partially compactify $(M, g)$ and then minimize the brane action in a suitable homology class.

To partially compactify, we use the fact that the translations $x^{i} \rightarrow x^{i}+x_{0}^{i}$ are isometries on $\left(\mathbb{R}^{n+1}, g_{0}\right)$. Choosing $a>0$ sufficiently large, we can enclose the compact set $K$ in an infinitely long rectangular box, with sides determined by the "planes", $x^{i}= \pm a, i=1, \ldots, n,-\infty<t<\infty$. We can then identify points on opposite sides, $x^{i}=-a, x^{i}=a, i=1, \ldots, n$, of the box in the obvious manner to obtain an identification space which we denote by $(\hat{M}, \hat{g})$. Note that outside the compact set $K$,

$$
\hat{M}=\mathbb{R} \times T^{n}, \quad \hat{g}=d t^{2}+e^{2 t} h,
$$

where $h$ is a flat metric on the torus $T^{n}$. Thus, $(\hat{M}, \hat{g})$ is just a standard hyperbolic cusp outside the compact set $K$, with scalar curvature satisfying $S[\hat{g}] \geq-n(n+1)$ globally.

Choose $b>0$ large so that $K$ is contained in the region of $\hat{M}$ bounded between the toroidal slices $t= \pm b$, and fix a $t$-slice $\Sigma_{0}=\left\{t_{0}\right\} \times T^{n}, t_{0}>b . \Sigma_{0}$ separates $\hat{M}$ into an "inside" and an "outside", the inside being the component of $\hat{M}-\Sigma_{0}$ containing the cusp end $t=-\infty$. We consider the brane action of hypersurfaces $\Sigma$ homologous to $\Sigma_{0}$,

$$
\mathcal{B}(\Sigma)=A(\Sigma)-n V(\Sigma) .
$$

We note, as is needed to define $\mathcal{B}(\Sigma)$ unambiguously, that since $\Sigma$ is homologous to $\Sigma_{0}$ it, too, has a distinguished "inside" and "outside", determined by the fact that both $\Sigma_{0}$ and $\Sigma$ are homologous to a $t$-slice far out on the cusp end.

We now want to minimize the brane action $\mathcal{B}$ in the homology class $\left[\Sigma_{0}\right]$. The basic approach is to consider a minimizing sequence $\Sigma_{1}, \Sigma_{2}, \ldots$ and use the compactness results of geometric measure theory to extract a regular limit surface. The potential difficulty with this approach is that, in principle, the surfaces 


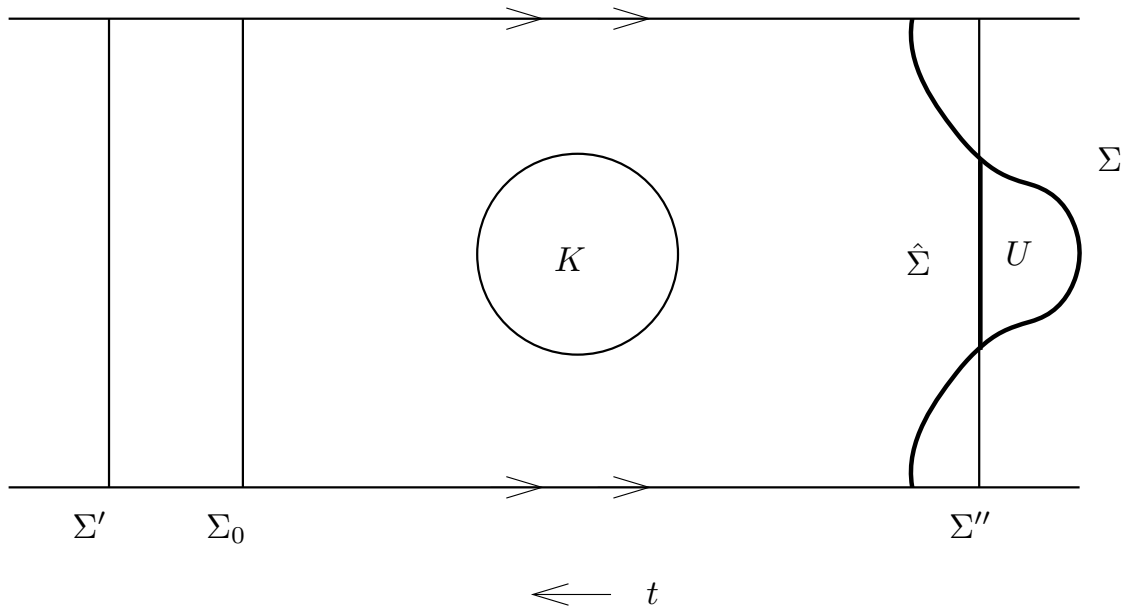

Figure 1. Replacing $\Sigma$ by $\hat{\Sigma}$.

$\Sigma_{1}, \Sigma_{2}, \ldots$, or portions of them, may drift out to infinity along either end of $\hat{M}$. But, in fact, that can be avoided in the present situation, owing to the existence of natural barrier surfaces, namely the $t$-slices themselves.

Fix $t$-slices $\Sigma^{\prime}=\left\{t_{1}\right\} \times \Sigma, t_{1}>t_{0}$, and $\Sigma^{\prime \prime}=\left\{t_{2}\right\} \times \Sigma, t_{2}<-b$. We show that any minimizing sequence can be replaced by a minimizing sequence contained in the region between $\Sigma^{\prime}$ and $\Sigma^{\prime \prime}$. To this end, consider a hypersurface $\Sigma$ homologous to $\Sigma_{0}$ that extends beyond $\Sigma^{\prime \prime}$ into the region $t<t_{2}$. Without loss of generality we may assume $\Sigma$ meets $\Sigma^{\prime \prime}$ transversely. Let $D$ be the part of $\Sigma$ meeting $\left\{t \leq t_{2}\right\}$, and let $U$ be the domain bounded by $\Sigma^{\prime \prime}$ and $D$. Then $\partial U$ consists of $D$ and a part $D^{\prime \prime}$ of $\Sigma^{\prime \prime}$. Let $\hat{\Sigma}$ be the hypersurface homologous to $\Sigma_{0}$ obtained from $\Sigma$ by replacing $D$ with $D^{\prime \prime}$ (see Figure 2).

Since $U$ is contained in a region where the metric (2.16) applies, and since in this region $\operatorname{div}\left(\partial_{t}\right)=n$, we apply the divergence theorem to obtain,

$$
\begin{aligned}
n \mathcal{V}(\hat{\Sigma})-n \mathcal{V}(\Sigma) & =n \operatorname{vol}(U)=\int_{U} \operatorname{div}\left(\partial_{t}\right) d V \\
& =\int_{D}\left\langle\partial_{t}, n\right\rangle d A+\int_{D^{\prime \prime}}\left\langle\partial_{t}, \partial_{t}\right\rangle d A \\
& \geq-A(D)+A\left(D^{\prime \prime}\right)=-A(\Sigma)+A(\hat{\Sigma}) .
\end{aligned}
$$

Rearranging this inequality gives the desired, $\mathcal{B}(\hat{\Sigma}) \leq \mathcal{B}(\Sigma)$. By a similar argument the same conclusion holds if $\Sigma$ extends beyond $\Sigma^{\prime}$ into the region $\left\{t>t_{1}\right\}$.

Thus, we can choose a minimizing sequence $\Sigma_{i}$, for the brane action within the homology class $\left[\Sigma_{0}\right]$ that is confined to the compact region between $\Sigma^{\prime}$ and $\Sigma^{\prime \prime}$. Since $\mathcal{V}\left(\Sigma_{i}\right) \leq \mathcal{V}\left(\Sigma^{\prime}\right)$, we are ensured that $\lim _{i \rightarrow \infty} \mathcal{B}\left(\Sigma_{i}\right)>-\infty$. Then the compactness and regularity results of geometric measure theory (see, e.g., [14,22] and 
references therein) guarantee the existence of a regular embedded hypersurface $S$ homologous to $\Sigma_{0}$ that achieves a minimum of the brane action on $\left[\Sigma_{0}\right]$. In general $S$ will be a sum of connected embedded surfaces, $S=S_{1}+\cdots+S_{n}$.

The next thing we wish to observe is that there is a nonzero degree map from $S$ to the $n$-torus $\Sigma_{0}$. This map comes from the 'almost product' structure of $\hat{M}$ given in (2.10). A simple deformation of the $t$-lines in the vicinity of $K$ can be used to produce a continuous projection type map $P: \hat{M} \rightarrow \Sigma_{0}$ such that $K$ gets sent to a single point on $\Sigma_{0}$ under $P$, and such that $P \circ j=$ id, where $j: \Sigma_{0} \rightarrow \hat{M}$ is inclusion. Then $f=P \circ i: S \rightarrow \Sigma_{0}$, where $i: S \rightarrow \hat{M}$ is inclusion, is the desired nonzero degree map. Indeed, $f$ induces the map on homology $f_{*}: H_{n}(S) \rightarrow H_{n}\left(\Sigma_{0}\right)$, and using that $S$ is homologous to $\Sigma_{0}$, we compute, $f_{*}[S]=P_{*}\left(i_{*}[S]\right)=P_{*}\left(j_{*}\left[\Sigma_{0}\right]\right)=\operatorname{id}_{*}\left[\Sigma_{0}\right]=\Sigma_{0} \neq 0$.

Thus, by linearity of $f_{*}$, at least one of the components of $S, S_{1}$, say, admits a nonzero degree map to the $n$-torus. By a result of Schoen and Yau [22], which does not require a spin assumption, $S_{1}$ does not admit a metric of positive scalar curvature. (In fact it admits a metric of nonnegative scalar curvature only if it is flat). Moreover, we know that $S_{1}$ minimizes the brane action in its homology class (otherwise there would exist a hypersurface homologous to $\Sigma_{0}$ with brane action strictly less than that of $S$ ). Thus, we can apply Theorem 2.3 to conclude that a neighborhood $U$ of $S_{1}$ splits as a warped product,

$$
U=\left(-u_{0}, u_{0}\right) \times\left. S_{1} \quad \hat{g}\right|_{U}=d u^{2}+e^{2 u} h,
$$

where the induced metric $h$ on $S_{1}$ is flat. But since $S_{1}$ in fact globally maximizes the brane action in its homology class, by standard arguments this local warped product structure can be extended to arbitrarily large $u$-intervals. Hence $K$ will eventually be contained in this constructed warp product region. It now follows that $\hat{M}$ has constant curvature $K_{\hat{M}}=-1$. This in turn implies that $M$ has constant curvature $K_{M}=-1$. By previous remarks, we conclude that $M$ is globally isometric to hyperbolic space.

\section{Positivity of mass}

The aim of this section is to give a proof of Theorem 1.3 on the positivity of mass in the asymptotically hyperbolic setting. We shall adopt here the definition of asymptotically hyperbolic given in Wang [26]:

Definition 3.1. A Riemannian manifold $\left(M^{n+1}, g\right)$ is asymptotically hyperbolic provided it is conformally compact, with smooth conformal compactification $(\tilde{M}, \tilde{g})$, and with conformal boundary $\partial \tilde{M}=S^{n}$, such that the metric $g$ on a deleted neighborhood $(0, T) \times S^{n}$ of $\partial \tilde{M}=\{t=0\}$ takes the form

$$
g=\sinh ^{-2}(t)\left(d t^{2}+h\right),
$$


where $h=h(t, \cdot)$ is a family of metrics on $S^{n}$, depending smoothly on $t \in[0, T)$, of the form,

$$
h=h_{0}+t^{n+1} k+O\left(t^{n+2}\right),
$$

where $h_{0}$ is the standard metric on $S^{n}$, and $k$ is a symmetric 2-tensor on $S^{n}$.

We refer to $k$ as the mass aspect tensor; it is the leading order measure of the deviation of the metric $g$ from the hyperbolic metric. Its trace with respect to $h_{0}, \operatorname{tr}_{h_{0}} k$, is called the mass aspect function. Up to a normalizing constant, the integral of the mass aspect function over the sphere defines the mass (or energy) of $(M, g)$, mass $=\int_{S^{n}} \operatorname{tr}_{h_{0}} k$.

For convenience, we repeat here the statement of our positivity of mass result.

Theorem 3.1. Let $\left(M^{n+1}, g\right), 2 \leq n \leq 6$, be an asymptotically hyperbolic manifold with scalar curvature $S[g] \geq-n(n+1)$. Assume that the mass aspect function $t_{h_{0}} k$ does not change sign, i.e., that it is either negative, zero, or positive. Then, either the mass of $(M, g)$ is positive, or $(M, g)$ is isometric to hyperbolic space.

The proof, which makes use of the rigidity result Theorem 1.1, is carried out in the next two subsections. In Subsection 3.1 we obtain the deformation result mentioned in the introduction, see Theorem 3.2 below. This, together with Theorem 1.1, implies that the mass aspect function cannot be negative, see Proposition 3.3. In Subsection 3.2, it is proved, using Theorem 1.1 again, that if the mass aspect function vanishes then $(M, g)$ is isometric to hyperbolic space, see Theorem 3.9. These results together imply Theorem 3.1.

\subsection{The deformation result}

Suppose $\left(M^{n+1}, g\right)$ is asymptotically hyperbolic in the sense of Definition 3.1. Then by making the change of coordinate, $t=\operatorname{arcsinh}\left(\frac{1}{r}\right)$, in (3.19) it follows that there is a relatively compact set $K$ such that $M \backslash K=S^{n} \times[R, \infty), R>0$, and on $M \backslash K, g$ has the form,

$$
g=\frac{1}{1+r^{2}} d r^{2}+r^{2} h
$$

where $h=h(\cdot, r)$ is an $r$-dependent family of metrics on $S^{n}$ of the form,

$$
h=h_{0}+\frac{1}{r^{n+1}} k+\sigma,
$$

where $h_{0}$ is the standard metric on $S^{n}, k$ is the mass aspect tensor and $\sigma=\sigma(\cdot, r)$ is an $r$-dependent family of metrics on $S^{n}$ such that for integers $\ell, m \geq 0$, one has,

$$
\left|\left(r \partial_{r}\right)^{\ell} \partial_{x}^{m} \sigma\right| \leq C / r^{n+2},
$$

for some constant $C$. For the proof of the deformation theorem and the positive mass theorem, it is sufficient to assume condition (3.23) for $0 \leq \ell, m \leq 2$.

Let $\left(M^{n+1}, g\right)$ be asymptotically hyperbolic, with scalar curvature satisfying, $S[g] \geq-n(n+1)$. What we now prove is that if the mass aspect function of $(M, g)$ is pointwise negative then $g$ can be deformed on an arbitrarily small neighborhood of infinity to the hyperbolic metric, while preserving (after a change of scale) the 
scalar curvature inequality $S \geq-n(n+1)$. A more precise statement is given below.

Theorem 3.2. Let the metric $g$ be given as above. Suppose that the scalar curvature of $g, S[g]$, satisfies $S[g] \geq-n(n+1)$. If the mass aspect function $t_{h_{0}} k$ is pointwise negative, then for any sufficiently large $R_{1}>R$ there exists a metric $\hat{g}$ on $M$ such that,

$$
\hat{g}= \begin{cases}g, & R \leq r \leq R_{1} \\ g_{a}=\frac{1}{1+\frac{r^{2}}{a}} d r^{2}+r^{2} h_{0}, & 9 \lambda R_{1} \leq r<\infty,\end{cases}
$$

where $a \in(0,1)$, and such that,

$$
S[\hat{g}] \geq-\frac{n(n+1)}{a} .
$$

(The constant $\lambda>1$ depends only on the mass aspect function; see Section 3.1.1.)

Theorems 1.1 and 3.2 may be combined to give the following result.

Proposition 3.3. Let $\left(M^{n+1}, g\right), 2 \leq n \leq 6$, be an asymptotically hyperbolic manifold with scalar curvature satisfying, $S[g] \geq-n(n+1)$. Then the mass aspect function $\operatorname{tr}_{h_{0}} k$ cannot be everywhere pointwise negative.

Proof. Suppose to the contrary that the mass aspect function is strictly negative. Given any $p \in M$, choose $R$ large enough so that $p$ is not in the end $S^{n} \times[R, \infty)$. Theorem 3.2, together with a rescaling of the metric, implies the existence of a metric $\tilde{g}$ on $M$ such that $(M, \tilde{g})$ satisfies the hypotheses of Theorem 1.1. Hence, $(M, \tilde{g})$ is isometric to hyperbolic space $\mathbb{H}^{n+1}$. But, modulo the change of scale, by our construction, $\tilde{g}$ will differ from $g$ only at points on the end $S^{n} \times[R, \infty)$. It follows that $\left(M^{n+1}, g\right)$ has constant negative curvature curvature in a neighborhood of $p$. Since $p$ is arbitrary, $\left(M^{n+1}, g\right)$ must have globally constant negative curvature, which, by the asymptotics of $\left(M^{n+1}, g\right)$, must equal -1 . Since $M$ is simply connected at infinity, we conclude that $\left(M^{n+1}, g\right)$ is isometric to hyperbolic space $\mathbb{H}^{n}$. But this contradicts the assumption that the mass aspect function is negative.

Proof of Theorem 3.2. We now turn to the proof of the deformation result. Introduce coordinates $x=\left(x^{1}, x^{2}, \ldots, x^{n}\right)$ on $S^{n}$. We use the convention that for a function $f=f(x, r), f^{\prime}(x, r)=\partial_{r} f(x, r)$, and $f^{\prime \prime}(x, r)=\partial_{r}^{2} f(x, r)$.

Let $\omega_{i j}$ and $k_{i j}$ be the components of $h_{0}$ and $k$, respectively, with respect to the coordinates $\left(x^{1}, x^{2}, \ldots, x^{n}\right)$. Then $g$ in $(3.21)$ takes the form

$$
g=\frac{d r^{2}}{1+r^{2}}+r^{2}\left(\omega_{i j}+\frac{\alpha_{i j}}{r^{n+1}}\right) d x^{i} d x^{j}
$$

where

$$
\alpha_{i j}=k_{i j}(x)+\frac{\beta_{i j}(x, r)}{r} .
$$


We are assuming (cf., (3.23)) $\alpha_{i j}$ satisfies the bounds,

$$
\left|\left(r \partial_{r}\right)^{\ell} \partial_{x}^{m} \alpha_{i j}\right| \leq \Lambda \text {. }
$$

for all integers $\ell, m, 0 \leq \ell, m \leq 2$.

Let $\mu$ denote the the mass aspect function, $\mu=\operatorname{tr}_{h_{0}} k=\omega^{i j} k_{i j}$; by assumption, $|\mu|>0$. Let $\bar{\mu}=\max _{x}|\mu(x)|, \mu=\min _{x}|\mu(x)|$. We shall be making estimates of geometric quantities in terms of the above defined constants. In particular, we shall use a generic constant

$$
C=C(n, R, \Lambda, \bar{\mu}, \underline{\mu})
$$

depending only on $n, R, \Lambda, \bar{\mu}, \underline{\mu}$, which may change from line to line. We shall further use the notation $O\left(1 / r^{\bar{k}}\right)$ for a quantity bounded by $C(n, R, \Lambda, \bar{\mu}, \underline{\mu}) / r^{k}$.

3.1.1. Preliminary definitions. Fix $R_{1}>R$ to be specified later. Set

$$
\lambda=\left(\frac{\bar{\mu}}{\underline{\mu}}\right)^{\frac{1}{n+1}} \text {. }
$$

Let $a=a\left(n, \bar{\mu}, \underline{\mu}, R_{1}\right) \in(0,1)$ be a number such that

$$
\frac{n+1}{n} \sqrt{\frac{4}{3}} \frac{\bar{\mu}}{\left(4 \lambda R_{1}\right)^{n+1}}<\frac{1}{a}-1<\frac{n+1}{n} \sqrt{\frac{3}{4}} \frac{\underline{\mu}}{\left(3 \lambda R_{1}\right)^{n+1}} .
$$

To show such an $a$ exists, it suffices to show that

$$
\frac{n+1}{n} \sqrt{\frac{4}{3}} \frac{\bar{\mu}}{\left(4 \lambda R_{1}\right)^{n+1}}<\frac{n+1}{n} \sqrt{\frac{3}{4}} \frac{\underline{\mu}}{\left(3 \lambda R_{1}\right)^{n+1}}
$$

or equivalently

$$
\frac{3}{4}\left(\frac{4}{3} \lambda\right)^{n+1}>\frac{\bar{\mu}}{\underline{\mu}}
$$

By our choice of $\lambda$, this is equivalent to

$$
\left(\frac{4}{3}\right)^{n}>1
$$

which is obviously true. It follows from the definition that $a \nearrow 1$ as $R_{1} \nearrow \infty$.

It is straightforward to show the existence of a smooth function $\psi: \mathbb{R} \rightarrow \mathbb{R}_{+}$ such that for any $R_{1}>R$.

$$
\begin{aligned}
\psi & = \begin{cases}1, & r \leq 7 \lambda R_{1} \\
0, & r \geq 8 \lambda R_{1}\end{cases} \\
\psi^{\prime}(r) & \leq 0 \quad \text { for all } r \\
\left|\psi^{\prime}(r)\right| & \leq \frac{b}{r} \\
\left|\psi^{\prime \prime}(r)\right| & \leq \frac{c}{r^{2}}
\end{aligned}
$$

where $b, c$ are positive constants. In the following, we consider a fixed function $\psi$ satisfying the conditions (3.27). 
In the deformation construction, we shall consider functions $f: S^{n} \times[R, \infty) \rightarrow$ $\mathbb{R}$, satisfying the following conditions.

$$
f(x, R)=1, \quad \text { for } \quad x \in S^{n},
$$

and for $(x, r) \in S^{n} \times[R, \infty)$ the conditions

$$
\begin{aligned}
\frac{1}{2} & \leq f \leq 2 \\
\left|f^{\prime}(x, r)\right| & \leq \frac{1}{r^{2}} \\
\left|\Delta_{S^{n}} f\right| & \leq \frac{1}{r^{n}} \\
\left|\nabla^{S^{n}} f\right|^{2} & \leq \frac{1}{r^{n}} .
\end{aligned}
$$

In particular, the constant function $f \equiv 1$ satisfies conditions (3.28). In order to carry out the deformation from the metric $g$ to the metric $g_{a}$, we shall consider metrics of the form,

$$
g_{f, \psi}=\frac{1}{1+r^{2} f} d r^{2}+r^{2}\left(\omega_{i j}+\frac{\psi \alpha_{i j}}{r^{n+1}}\right) d x^{i} d x^{j} .
$$

Given $\psi$, the main objective is to construct an $f$ satisfying (3.28) so that $g_{f, \psi}$ has the required properties.

3.1.2. Scalar curvature formulas. We need formulas for the scalar curvature $S\left[g_{f, \psi}\right]$ of the metric $g_{f, \psi}$.

Lemma 3.4. Let $f, \psi$ satisfy the assumptions (3.28) and (3.27). Then the metric $g_{f, \psi}$ has scalar curvature,

$$
S\left[g_{f, \psi}\right]=-n(n+1) f-n r f^{\prime}+\frac{1}{r^{n}}\left(n|\mu| \psi^{\prime}-r|\mu| \psi^{\prime \prime}\right) f+J,
$$

where $J$ is a term bounded by $C(n, R, \Lambda, \bar{\mu}, \mu) / r^{n+2}$, and such that $J=0$ for $r \geq 8 \lambda R_{1}$.

Proof. We describe our approach to carrying out this computation. Setting,

$$
h=1+r^{2} f \quad \text { and } \quad g_{i j}=r^{2}\left(\omega_{i j}+\frac{\psi \alpha_{i j}}{r^{n+1}}\right),
$$

$g_{f, \psi}$ becomes,

$$
g_{f, \psi}=\frac{1}{h} d r^{2}+g_{i j} d x^{i} d x^{j} .
$$

Applying the Gauss equation to an $r$-slice $\Sigma=S^{n} \times\{r\}$ gives,

$$
S\left[g_{f, \psi}\right]=S_{\Sigma}+|B|^{2}-H^{2}+2 \operatorname{Ric}(N, N),
$$

where $S_{\Sigma}, B$ and $H$ are the scalar curvature, second fundamental form and mean curvature of $\Sigma$, respectively, and $\operatorname{Ric}(N, N)$ is the ambient Ricci curvature in the direction of the unit normal $N=h^{1 / 2} \frac{\partial}{\partial r}$. In terms of coordinates, $B$ and $H$ are 
given by, $b_{i j}=B\left(\partial_{i}, \partial_{j}\right)=\frac{1}{2} \sqrt{h} \partial_{r} g_{i j}$, and $H=g^{i j} b_{i j}$. We then compute each term in (3.33) in turn.

For the first three terms we obtain, making use of the bounds (3.26) and (3.27)

$$
\begin{aligned}
S_{\Sigma} & =\frac{n(n-1)}{r^{2}}+O\left(\frac{1}{r^{n+3}}\right) \\
H & =h^{\frac{1}{2}}\left[\frac{n}{r}+\frac{n+1}{2} \frac{|\mu| \psi}{r^{n+2}}-\frac{1}{2} \frac{|\mu| \psi^{\prime}}{r^{n+1}}+O\left(\frac{1}{r^{n+3}}\right)\right] \\
|B|^{2} & =B_{i}{ }^{j} B_{j}{ }^{i}=h\left[\frac{n}{r^{2}}+(n+1) \frac{|\mu| \psi}{r^{n+3}}-\frac{|\mu| \psi^{\prime}}{r^{n+2}}+O\left(\frac{1}{r^{n+4}}\right)\right] .
\end{aligned}
$$

Equations (3.35) and (3.36) combine to give,

$$
\begin{aligned}
|B|^{2}-H^{2}= & h\left[-\frac{n(n-1)}{r^{2}}-(n-1)(n+1) \frac{|\mu| \psi}{r^{n+3}}\right. \\
& \left.+(n-1) \frac{|\mu| \psi^{\prime}}{r^{n+2}}+O\left(\frac{1}{r^{n+4}}\right)\right] .
\end{aligned}
$$

Applying the Raychaudhuri equation to the unit normal $N=h^{1 / 2} \frac{\partial}{\partial r}$ to the level sets of $r$, we have,

$$
\operatorname{Ric}(N, N)=-N(H)-|B|^{2}-\sqrt{h} \Delta_{\Sigma} \frac{1}{\sqrt{h}} .
$$

Making use of equations (3.35) and (3.36), we derive from (3.38),

$$
\begin{aligned}
\operatorname{Ric}(N, N)= & -\frac{1}{2} h^{\prime}\left[\frac{n}{r}+\frac{n+1}{2} \frac{|\mu| \psi}{r^{n+2}}-\frac{1}{2} \frac{|\mu| \psi^{\prime}}{r^{n+1}}+O\left(\frac{1}{r^{n+3}}\right)\right] \\
& +h\left[\frac{n(n+1)}{2} \frac{|\mu| \psi}{r^{n+3}}-n \frac{|\mu| \psi^{\prime}}{r^{n+2}}+\frac{1}{2} \frac{|\mu| \psi^{\prime \prime}}{r^{n+1}}+O\left(\frac{1}{r^{n+4}}\right)\right] \\
& -\sqrt{h} \Delta_{\Sigma} \frac{1}{\sqrt{h}} .
\end{aligned}
$$

Equations (3.34), (3.37) and (3.39) then combine to give,

$$
\begin{aligned}
S\left[g_{f, \psi}\right]= & \frac{n(n-1)}{r^{2}}-\frac{n(n-1)}{r^{2}} h-\frac{n}{r} h^{\prime}-\left[\frac{n+1}{2} \frac{|\mu| \psi}{r^{n+2}}-\frac{1}{2} \frac{|\mu| \psi^{\prime}}{r^{n+1}}+O\left(\frac{1}{r^{n+3}}\right)\right] h^{\prime} \\
& +\left[(n+1) \frac{|\mu| \psi}{r^{n+3}}-(n+1) \frac{|\mu| \psi^{\prime}}{r^{n+2}}+\frac{|\mu| \psi^{\prime \prime}}{r^{n+1}}+O\left(\frac{1}{r^{n+4}}\right)\right] h \\
& -2 \sqrt{h} \triangle_{\Sigma} \frac{1}{\sqrt{h}}+O\left(\frac{1}{r^{n+3}}\right) .
\end{aligned}
$$

Setting $h=1+r^{2} f$ in the above, and making use of the bounds (3.28) and (3.27), one derives in a straight forward manner equation (3.30). Moreover, it is clear from the computations that all 'big $\mathrm{O}$ ' terms vanish once $\psi$ vanishes.

The following Corollary gives the form of the scalar curvature which will be used in the deformation construction. 
Corollary 3.5. Let $f, \psi$ satisfy the assumptions (3.28) and (3.27). Then, there is a nonnegative function $A_{1}:[R, \infty) \rightarrow \mathbb{R}_{+}$, independent of $f$, such that

$$
A_{1} \leq C(n, R, \Lambda, \bar{\mu}, \underline{\mu}) \quad \text { and } \quad A_{1}=0 \quad \text { for } r \geq 9 \lambda R_{1},
$$

and such that the scalar curvature $S\left[g_{f, \psi}\right]$ of $g_{f, \psi}$ satisfies the inequality

$$
S\left[g_{f, \psi}\right] \geq-\frac{n}{r^{n}}\left[\left(r^{n+1}+\frac{n+1}{n}|\mu| \psi-\frac{r}{n}|\mu| \psi^{\prime}\right) f-\frac{A_{1}(r)}{r}\right]^{\prime} .
$$

Proof. Using the product rule, equation (3.30) may be expressed as,

$$
\begin{aligned}
S\left[g_{f, \psi}\right]= & -\frac{n}{r^{n}}\left(r^{n+1} f\right)^{\prime}+\frac{1}{r^{n}}\left[\left((n+1)|\mu| \psi-r|\mu| \psi^{\prime}\right) f\right]^{\prime} \\
& -\frac{1}{r^{n}}\left[(n+1)|\mu| \psi-r|\mu| \psi^{\prime}\right] f^{\prime}+O\left(\frac{1}{r^{n+2}}\right)
\end{aligned}
$$

which, by the bounds (3.28) and (3.27) simplifies to,

$$
S\left[g_{f, \psi}\right]=-\frac{n}{r^{n}}\left[\left(r^{n+1}-\frac{n+1}{n}|\mu| \psi+\frac{r}{n}|\mu| \psi^{\prime}\right) f\right]^{\prime}+O\left(\frac{1}{r^{n+2}}\right) .
$$

It follows that there exists a smooth function $A:[R, \infty) \rightarrow \mathbb{R}_{+}$, satisfying, $A \leq$ $C(n, R, \Lambda, \bar{\mu}, \underline{\mu})$ and $A=0$ for $r \geq 9 \lambda R_{1}$, such that,

$$
S\left[g_{f, \psi}\right] \geq-\frac{n}{r^{n}}\left[\left(r^{n+1}-\frac{n+1}{n}|\mu| \psi+\frac{r}{n}|\mu| \psi^{\prime}\right) f\right]^{\prime}-\frac{A(r)}{r^{n+2}} .
$$

Now define $A_{1}:[R, \infty) \rightarrow \mathbb{R}_{+}$by,

$$
A_{1}(r)=\frac{r}{n} \int_{r}^{9 \lambda R_{1}} \frac{A(t)}{t^{2}} d t .
$$

One easily checks that the properties (3.41) hold. Moreover, since,

$$
\left(\frac{A_{1}(r)}{r}\right)^{\prime}=-\frac{1}{n} \frac{A(r)}{r^{2}}
$$

inequality (3.42) follows from (3.44). Finally, it is clear from the construction that $A$ and hence also $A_{1}$ may be chosen to be independent of $f$.

3.1.3. Defining $\eta$ : Rounding the corner. Let $\eta_{1}(x, r), \eta_{2}(r)$ be given by

$$
\begin{aligned}
\eta_{1}(x, r) & =r^{n+1}+\frac{n+1}{n}|\mu| \psi-\frac{r}{n}|\mu| \psi^{\prime}-\frac{A_{1}(r)}{r}, \\
\eta_{2}(r) & =\frac{r^{n+1}}{a},
\end{aligned}
$$

where the function $A_{1}$ appearing in $\eta_{1}$ is the function $A_{1}$ defined in Corollary 3.5. By (3.42), $S\left[g_{1, \psi}\right] \geq-\frac{n}{r^{n}} \eta_{1}^{\prime}$. Further, $g_{a}$ has constant curvature $-1 / a$, and hence $S\left[g_{a}\right]=-\frac{n}{r^{n}} \eta_{2}^{\prime}$.

In the rest of the argument we shall be choosing $R_{1}$ sufficiently large, so that the required conditions are satisfied. We shall successively increase $R_{1}$ as required. 




Figure 2. Construction of $\eta$.

Lemma 3.6. There is an $R_{1}>R, R_{1}=R_{1}(n, R, \Lambda, \bar{\mu}, \underline{\mu})$ such that the following inequalities hold.

$$
\begin{aligned}
\eta_{1}^{\prime}(x, r) & <\eta_{2}^{\prime}(r), & R_{1} & \leq r \leq 7 \lambda R_{1} \\
\eta_{1}(x, r)-\eta_{2}(r) & >\underline{\mu} / 20, & R_{1} & \leq r \leq 3 \lambda R_{1} \\
\eta_{2}(r)-\eta_{1}(x, r) & >\bar{\mu} / 10, & 4 \lambda R_{1} & \leq r \leq 7 \lambda R_{1} .
\end{aligned}
$$

Proof. We need to consider only the interval $R_{1} \leq r \leq 7 \lambda R_{1}$. There, $\psi \equiv 1$, and $\psi^{\prime} \equiv 0$. We start by proving (3.48a). By construction, we have for $r \geq R_{1}$, using $|\mu|>0$ and the properties of $a$, after some manipulations,

$$
\begin{aligned}
\eta_{2}^{\prime}-\eta_{1}^{\prime}>\frac{1}{r}\left[\left(\frac{1}{a}-1\right)(n+1) r^{n+1}-\frac{A(r)}{n r}\right] \\
>\frac{1}{r}\left[\sqrt{\frac{4}{3}} \frac{(n+1)^{2}}{n} \frac{\bar{\mu}}{(4 \lambda)^{n+1}}-\frac{A(r)}{n r}\right] .
\end{aligned}
$$

Since $A(r) \leq C(n, \Lambda, R, \bar{\mu}, \underline{\mu})$, it follows that (3.48a) holds for $R_{1}$ sufficiently large.

Next we prove $(3.48 \mathrm{~b})$. Since $\left(\eta_{1}-\eta_{2}\right)^{\prime}<0$ for $r \geq R_{1}$, it is sufficient to prove the inequality at $r=3 \lambda R_{1}$. We have

$$
\begin{aligned}
\eta_{1}\left(x, 3 \lambda R_{1}\right)-\eta_{2}\left(3 \lambda R_{1}\right) \geq & {\left[\left(1-\frac{1}{a}\right)+\frac{n+1}{n} \frac{\underline{\mu}}{\left(3 \lambda R_{1}\right)^{n+1}}\right]\left(3 \lambda R_{1}\right)^{n+1} } \\
& -\frac{A_{1}\left(3 \lambda R_{1}\right)}{3 \lambda R_{1}},
\end{aligned}
$$

which by using the properties of $a$ and simplifying gives,

$$
>\left[1-\sqrt{\frac{3}{4}}\right] \frac{n+1}{n} \underline{\mu}-\frac{A_{1}\left(3 \lambda R_{1}\right)}{3 \lambda R_{1}} .
$$


One checks that $1-\sqrt{3 / 4}>1 / 10$. Thus, in view of the fact that $A_{1} \leq C(n, R, \Lambda$, $\bar{\mu}, \mu)$, by possibly increasing $R_{1}$ we see that $(3.48 \mathrm{~b})$ can be made to hold.

We proceed in a similar fashion to prove $(3.48 \mathrm{c})$. We have

$$
\begin{aligned}
\eta_{2}\left(4 \lambda R_{1}\right)-\eta_{1}\left(x, 4 \lambda R_{1}\right) & \geq\left[\left(\frac{1}{a}-1\right)-\frac{n+1}{n} \frac{\bar{\mu}}{\left(4 \lambda R_{1}\right)^{n+1}}\right]\left(4 \lambda R_{1}\right)^{n+1} \\
& \geq\left[\sqrt{\frac{4}{3}}-1\right] \frac{n+1}{n} \bar{\mu} .
\end{aligned}
$$

We note that $\sqrt{4 / 3}-1>1 / 10$. This completes the proof of Lemma 3.6.

We shall define $\eta=\eta(x, r)$ to be a suitably increasing function of the variable $r$ that smoothly transitions from $\eta_{1}$ to $\eta_{2}$ (see Figure 1 ). In order to make meaningful estimates, its construction shall be made fairly explicit. Its construction depends on two auxiliary functions $\alpha$ and $\beta$, which we now introduce.

Let $\alpha:[0, \infty) \rightarrow \mathbb{R}$ be a function satisfying,

1. $\alpha(r)=0$ for $r \leq R_{1}, 2 R_{1} \leq r \leq 5 \lambda R_{1}$, and $r \geq 6 \lambda R_{1}$.

2. $\alpha>0$ for $R_{1}<r<2 R_{1}, \alpha<0$ for $5 \lambda R_{1}<r<6 \lambda R_{1}$, and

$$
\int_{R_{1}}^{6 \lambda R_{1}} \alpha(t) d t=\int_{-\infty}^{\infty} \alpha(t) d t=0 .
$$

Consider,

$$
\gamma(x, r)=\frac{\alpha(r)}{\eta_{1}(x, r)-\eta_{2}(r)} .
$$

It follows from Lemma 3.6 and the properties of $\alpha$ that $\gamma$ is nonnegative and bounded,

$$
0 \leq \gamma \leq C(n, \Lambda, \underline{\mu}, \bar{\mu})
$$

Next, define $m(x)$ by the condition

$$
1+m(x) \int_{R_{1}}^{6 \lambda R_{1}} \gamma(x, t) d t=0,
$$

and let

$$
\beta(x, r)=1+m(x) \int_{R_{1}}^{r} \gamma(x, t) d t .
$$

Then, $\beta$ satisfies,

$$
\begin{aligned}
\beta & =1, & & \text { for } r \leq R_{1} \\
\beta & =0, & & \text { for } r \geq 6 \lambda R_{1} \\
0 \leq \beta & \leq 1, & & \text { for all } r .
\end{aligned}
$$

Conditions (3.51a), (3.51b) are clear. For (3.51c), we consider, $\beta^{\prime}=m \gamma$. As observed above, $\gamma \geq 0$, and hence from (3.50), $m \leq 0$, so that $m \gamma \leq 0$. Hence $\beta$ is decreasing, which implies that $0 \leq \beta \leq 1$. 
Now we are ready to define $\eta$. Let

$$
\eta(x, r)=\eta_{1}\left(x, R_{1}\right)+\int_{R_{1}}^{r}\left[\beta(x, t) \eta_{1}^{\prime}(x, t)+(1-\beta(x, t)) \eta_{2}^{\prime}(t)\right] d t .
$$

Lemma 3.7. $\eta$ defined as above satisfies the conditions

$$
\begin{gathered}
\eta(x, r)= \begin{cases}\eta_{1}(x, r) & r \leq R_{1} \\
\eta_{2}(r)=\frac{r^{n+1}}{a}, & r \geq 6 \lambda R_{1}\end{cases} \\
\eta^{\prime}(x, r) \leq \eta_{2}^{\prime}(r)=\frac{(n+1) r^{n}}{a}, \quad R_{1} \leq r<\infty .
\end{gathered}
$$

Proof. For $r \leq R_{1}$, we have $\beta=1$, so

$$
\eta(x, r)=\eta_{1}\left(x, R_{1}\right)+\int_{R_{1}}^{r} \eta_{1}^{\prime}(x, t) d t=\eta_{1}(x, r) .
$$

In the following calculations, which only involve derivatives and integrals with respect to $r$, we suppress reference to $x$ in order to avoid clutter. For $r \geq 6 \lambda R_{1}$, we have $\beta=0$, which gives

$$
\begin{aligned}
\eta(r) & =\eta_{1}\left(R_{1}\right)+\int_{R_{1}}^{6 \lambda R_{1}}\left[\beta \eta_{1}^{\prime}+(1-\beta) \eta_{2}^{\prime}\right] d t+\int_{6 \lambda R_{1}}^{r} \eta_{2}^{\prime} d t \\
& =\eta_{2}(r)-\eta_{2}\left(6 \lambda R_{1}\right)+\eta_{1}\left(R_{1}\right)+\int_{R_{1}}^{6 \lambda R_{1}}\left(\beta \eta_{1}^{\prime}+(1-\beta) \eta_{2}^{\prime}\right) d t
\end{aligned}
$$

A partial integration gives

$$
\begin{aligned}
& \int_{R_{1}}^{6 \lambda R_{1}}\left(\beta \eta_{1}^{\prime}+(1-\beta) \eta_{2}^{\prime}\right) d t \\
& \quad=\eta_{2}\left(6 \lambda R_{1}\right)-\eta_{2}\left(R_{1}\right)+\left.\beta\left(\eta_{1}-\eta_{2}\right)\right|_{r=R_{1}} ^{r=6 \lambda R_{1}}-\int_{R_{1}}^{6 \lambda R_{1}} \beta^{\prime}\left(\eta_{1}-\eta_{2}\right) d t
\end{aligned}
$$

use the properties of $\beta$ and $\gamma$

$$
=\eta_{2}\left(6 \lambda R_{1}\right)-\eta_{2}\left(R_{1}\right)-\left(\eta_{1}\left(R_{1}\right)-\eta_{2}\left(R_{1}\right)\right)-m \int_{R_{1}}^{6 \lambda R_{1}} \alpha d t
$$

use (3.49)

$$
=\eta_{2}\left(6 \lambda R_{1}\right)-\eta_{1}\left(R_{1}\right) .
$$

Substituting this into the formula for $\eta(x, r)$, we obtain,

$$
\eta(x, r)=\eta_{2}(r), \quad \text { for } \quad r \geq 6 \lambda R_{1} .
$$

We have now established (3.52a). Next we prove (3.52b). We have

$$
\eta^{\prime}=\beta \eta_{1}^{\prime}+(1-\beta) \eta_{2}^{\prime}
$$


and hence, by (3.48),

$$
\begin{aligned}
\eta^{\prime} & \leq \beta \eta_{2}^{\prime}+(1-\beta) \eta_{2}^{\prime} \\
& =\eta_{2}^{\prime}=\frac{(n+1) r^{n}}{a} .
\end{aligned}
$$

We shall now make use of the function $\eta$ defined above to define a function $f$, which shall be shown to satisfy the conditions (3.28). This fact allows us to apply the result of Corollary 3.5 to estimate the scalar curvature of the deformed metric $g_{f, \psi}$ defined in terms of this $f$.

3.1.4. Defining $f$. We define $f$ by,

$$
f=\frac{\eta+\frac{A_{1}}{r}}{\eta_{1}+\frac{A_{1}}{r}}
$$

which implies,

$$
\left(r^{n+1}+\frac{n+1}{n}|\mu| \psi-\frac{r}{n}|\mu| \psi^{\prime}\right) f-\frac{A_{1}}{r}=\eta .
$$

Here, $A_{1}=A_{1}(r)$ is the function determined in Corollary 3.5. It is crucial to note here that $A_{1}$ is independent of the particular $f$, as long as it satisfies the conditions (3.28). Our task is now to show that $f$ defined as above does satisfy these conditions as long as $R_{1}$ is chosen sufficiently large. This will be demonstrated in Lemma 3.8 below. It then follows from equation (3.63) above, Corollary 3.5 and (3.52b) that $S\left[g_{f, \psi}\right]$ satisfies,

$$
S\left[g_{f, \psi}\right] \geq-\frac{n}{r^{n}} \eta^{\prime} \geq-\frac{n(n+1)}{a} .
$$

In addition we have, $g_{f, \psi}=g$ on $\left[R, R_{1}\right]$ and $g_{f, \psi}=g_{a}$ on $\left[9 \lambda R_{1}, \infty\right)$. Thus, subject to the following lemma, Theorem 3.2 has been proven.

Lemma 3.8. Let $\eta$ be as in Section 3.1.3, and let $f$ be given in terms of $\eta$ by (3.62). Then, there is an $R_{1}=R_{1}(n, R, \Lambda, \bar{\mu}, \underline{\mu})$ sufficiently large, so that the inequalities (3.28) are valid.

Proof. The condition (3.28a) is clear from the construction. Since (3.28c) implies (3.28b) we only need to verify that $\left|f^{\prime}\right| \leq 1 / r^{2}$ and $\left|\partial_{x} f\right| \leq 1 / r^{n},\left|\partial_{x}^{2} f\right| \leq$ $1 / r^{n}$. We begin by showing there is an $R_{1}$ sufficiently large, and not smaller than the previously made choices of $R_{1}$, so that $\left|f^{\prime}\right| \leq 1 / r^{2}$. We have,

$$
f=1+\frac{\left(\eta-\eta_{1}\right)}{\eta_{1}+A_{1} / r} \text {. }
$$

This gives

$$
f^{\prime}=\frac{\left(\eta-\eta_{1}\right)^{\prime}}{\eta_{1}+A_{1} / r}-\frac{\left(\eta-\eta_{1}\right)}{\left(\eta_{1}+A_{1} / r\right)^{2}}\left[\eta_{1}^{\prime}+\left(A_{1} / r\right)^{\prime}\right]
$$


Since

$$
\begin{aligned}
& \eta(x, r)=\eta_{1}\left(x, R_{1}\right)+\int_{R_{1}}^{r}\left(\beta \eta_{1}^{\prime}+(1-\beta) \eta_{2}^{\prime}\right) d t \\
& \eta_{1}(x, r)=\eta_{1}\left(x, R_{1}\right)+\int_{R_{1}}^{r} \eta_{1}^{\prime} d t
\end{aligned}
$$

we have,

$$
\eta-\eta_{1}=\int_{R_{1}}^{r}(1-\beta)\left(\eta_{2}-\eta_{1}\right)^{\prime} .
$$

so

$$
\left(\eta-\eta_{1}\right)^{\prime}=(1-\beta)\left(\eta_{2}-\eta_{1}\right)^{\prime}
$$

Hence,

$$
\begin{aligned}
\left|\left(\eta-\eta_{1}\right)^{\prime}\right| \leq\left(\eta_{2}-\eta_{1}\right)^{\prime} & =\left(\frac{1}{a}-1\right) r^{n}(n+1)+O(1 / r) \\
& \leq \frac{C}{r} .
\end{aligned}
$$

Here and below $C=C(n, R, \Lambda, \bar{\mu}, \underline{\mu})$ is a generic constant. Recall that by construction we have

$$
\left(A_{1} / r\right)^{\prime} \leq \frac{C}{r^{2}}
$$

Combining the above inequalities, we have

$$
\left|\frac{\left(\eta-\eta_{1}\right)^{\prime}}{\eta_{1}+A_{1} / r}\right| \leq \frac{C}{r^{n+2}} .
$$

Now since

$$
\left|\left(\eta-\eta_{1}\right)^{\prime}\right| \leq \frac{C}{r}
$$

we have

$$
\left|\eta-\eta_{1}\right| \leq C
$$

for $r \in\left[R_{1}, 9 \lambda R_{1}\right]$. This together with

$$
\left|\eta_{1}^{\prime}\right| \leq C r^{n}
$$

implies that

$$
\left|\frac{\left(\eta-\eta_{1}\right)}{\left(\eta_{1}+A_{1} / r\right)^{2}}\left[\eta_{1}^{\prime}+\left(A_{1} / r\right)^{\prime}\right]\right| \leq \frac{C}{r^{n+2}} .
$$

Equations (3.67) and (3.69) imply

$$
\left|f^{\prime}\right| \leq \frac{C}{r^{n+2}}
$$

for $r \in\left[R_{1}, 9 \lambda R_{1}\right]$. By choosing $R_{1}$ large enough, we have

$$
\left|f^{\prime}\right| \leq \frac{1}{r^{2}}
$$

which gives $(3.28 \mathrm{c})$. 
Next we demonstrate that by, if necessary, further increasing $R_{1}$, we can ensure that the condition

$$
\left|\partial_{x}^{k} f\right| \leq \frac{1}{r^{n}}, \quad k=1,2,
$$

holds, where $\partial_{x}$ denotes partial differentiation with respect to any one of the coordinates $x^{i}$.

Recalling (3.64), we have

$$
\partial_{x} f=\frac{\partial_{x}\left(\eta-\eta_{1}\right)}{\eta_{1}+A_{1} / r}-\frac{\eta-\eta_{1}}{\left(\eta_{1}+A_{1} / r\right)^{2}}\left(\partial_{x} \eta_{1}\right) .
$$

We estimate the second term first. By (3.68), $\left|\eta-\eta_{1}\right| \leq C$, so

$$
\left|\frac{\eta-\eta_{1}}{\left(\eta_{1}+A_{1} / r\right)^{2}} \partial_{x} \eta_{1}\right| \leq \frac{1}{r^{2 n+2}}\left|\partial_{x} \eta_{1}\right| .
$$

Recalling

$$
\eta_{1}=r^{n+1}+\frac{n+1}{n}|\mu| \psi-\frac{r}{n}|\mu| \psi^{\prime}-\frac{A_{1}}{r}
$$

we find $\left|\partial_{x} \eta_{1}\right| \leq C$. Hence the modulus of the second term in $\partial_{x} f$ is bounded by $C / r^{2 n+2}$.

Now consider the first term in $\partial_{x} f$. Recall

$$
\begin{aligned}
\eta-\eta_{1} & =\int_{R_{1}}^{r}(1-\beta)\left(\eta_{2}-\eta_{1}\right)^{\prime} d t \\
& =\left.(1-\beta)\left(\eta_{2}-\eta_{1}\right)\right|_{R_{1}} ^{r}+\int_{R_{1}}^{r}\left(\eta_{2}-\eta_{1}\right) \beta^{\prime} d t \\
& =[1-\beta]\left(\eta_{2}-\eta_{1}\right)+\int_{R_{1}}^{r}\left(\eta_{2}-\eta_{1}\right) \beta^{\prime} d t
\end{aligned}
$$

so

$$
\begin{aligned}
\partial_{x}\left(\eta-\eta_{1}\right)= & -\left(\partial_{x} \beta\right)\left(\eta_{2}-\eta_{1}\right)-(1-\beta) \partial_{x} \eta_{1} \\
& +\int_{R_{1}}^{r}\left(\left[\partial_{x}\left(\eta_{2}-\eta_{1}\right)\right] \beta^{\prime}+\left(\eta_{2}-\eta_{1}\right) \partial_{x} \beta^{\prime}\right) d t .
\end{aligned}
$$

Now,

$$
\partial_{x} \beta=\left(\partial_{x} m\right)\left(\int_{R_{1}}^{r} \frac{\alpha}{\eta_{1}-\eta_{2}}\right)+m \int_{R_{1}}^{r}\left(\partial_{x} \frac{\alpha}{\eta_{1}-\eta_{2}}\right) .
$$

Recall,

so

$$
1+m \int_{R_{1}}^{6 \lambda R_{1}} \frac{\alpha}{\eta_{1}-\eta_{2}}=0
$$

$$
m=-\frac{1}{\int_{R_{1}}^{6 \lambda R_{1}} \frac{\alpha}{\eta_{1}-\eta_{2}}}=O\left(1 / R_{1}\right) .
$$


Hence,

One can see that

$$
\partial_{x} m=-\frac{\int_{R_{1}}^{6 \lambda R_{1}} \partial_{x}\left(\frac{\alpha}{\eta_{1}-\eta_{2}}\right)}{\left(\int_{R_{1}}^{6 \lambda R_{1}} \frac{\alpha}{\eta_{1}-\eta_{2}}\right)^{2}} .
$$

$$
\partial_{x}\left(\frac{\alpha}{\eta_{1}-\eta_{2}}\right)=O(1),
$$

SO

$$
\partial_{x} m=O\left(1 / R_{1}\right) .
$$

Hence we have, using $m=O\left(1 / R_{1}\right)$,

$$
\partial_{x} \beta=O(1)
$$

and therefore

$$
\partial_{x} \beta\left(\eta_{2}-\eta_{1}\right)=O(1)
$$

Next we consider the second term in $\partial_{x} f$. We have

$$
\left|\partial_{x} \eta_{1}\right| \leq C
$$

So

$$
(1-\beta) \partial_{x} \eta_{1}=O(1)
$$

Since $m=O\left(1 / R_{1}\right)$, we have,

but,

$$
\beta^{\prime}=m \frac{\alpha}{\eta_{1}-\eta_{2}}=O\left(1 / R_{1}\right)
$$

$$
\partial_{x}\left(\eta_{2}-\eta_{1}\right)=O(1)
$$

so,

Similarly,

$$
\partial_{x}\left(\eta_{2}-\eta_{1}\right) \beta^{\prime}=O\left(1 / R_{1}\right)
$$

and

$$
\partial_{x} \beta^{\prime}=O\left(1 / R_{1}\right)
$$

$$
\eta_{2}-\eta_{1}=O(1)
$$

Hence,

$$
\left[\partial_{x}\left(\eta_{2}-\eta_{1}\right)\right] \beta^{\prime}+\left(\eta_{2}-\eta_{1}\right) \partial_{x} \beta^{\prime}=O\left(1 / R_{1}\right),
$$

and therefore,

$$
\int_{R_{1}}^{r}\left[\left(\partial_{x}\left(\eta_{2}-\eta_{1}\right)\right) \beta^{\prime}+\left(\eta_{2}-\eta_{1}\right) \partial_{x} \beta^{\prime}\right]=O(1) .
$$

Adding the three terms, we get,

$$
\partial_{x}\left(\eta-\eta_{1}\right)=O(1) .
$$

This implies that the first term in $\partial_{x} f$ is

$$
\frac{O(1)}{\eta_{1}+A_{1} / r}=O\left(1 / r^{n+1}\right)
$$


i.e.,

$$
\left|\partial_{x} f\right| \leq \frac{C}{r^{n+1}}
$$

Similar arguments give,

$$
\left|\partial_{x}^{2} f\right| \leq \frac{C}{r^{n+1}} .
$$

By choosing $R_{1}$ large enough we obtain,

$$
\left|\partial_{x}^{k} f\right| \leq \frac{1}{r^{n}}, \quad k=1,2 .
$$

Lemma 3.8 follows.

As discussed above, now that Lemma 3.8 is established, we have completed the proof of Theorem 3.2.

\subsection{The case of vanishing mass aspect function}

In this subsection, we prove the following.

Theorem 3.9. Let $\left(M^{n+1}, g\right), 2 \leq n \leq 6$, be an asymptotically hyperbolic manifold with scalar curvature satisfying, $S[g] \geq-n(n+1)$. If the mass aspect function $t_{h_{0}} k$ vanishes identically, then $(M, g)$ is isometric to hyperbolic space.

We note that while Theorem 3.9 generalizes Theorem 1.1, its proof relies on it. We note also that our positivity of mass result, Theorem 3.1, follows immediately from Proposition 3.3 and Theorem 3.9.

For notational convenience we set $d=n+1$. Further, let capital latin indices run from $1, \ldots, d-1$, let lowercase latin indices run from $1, \ldots, d$, and let $y^{A}$ be coordinates on $S^{d-1}$. Further, let $\left(x^{i}\right)=\left(t, y^{A}\right)$ be coordinates on $(0, T) \times S^{d-1}$, and, as usual, let $h_{0}$ be the standard metric on $S^{d-1}$.

3.2.1. Conformal gauge. Consider a conformally compact $d$-dimensional manifold $(M, g)$ where $M$ is the interior of a manifold with boundary $\tilde{M}=M \cup \partial M$, and suppose $g$ is of the form $g=\rho^{-2} \tilde{g}$, with $\rho$ a defining function for $\partial M$ for a metric $\tilde{g}$ which is smooth on $\tilde{M}$.

Let $\theta$ be a positive function on $\tilde{M}$. Letting $\tilde{g} \rightarrow \theta^{2} \tilde{g}$ and $\rho \rightarrow \theta \rho$ leaves $g$ unchanged. Such a transformation can therefore be viewed as a change of conformal gauge.

Let $\tilde{g}$ be a metric on $\tilde{M}$ which in a neighborhood of $\partial M$ can be written in the form

$$
\tilde{g}=d t^{2}+h_{0}+t^{d} \gamma
$$

where $\gamma=\gamma_{i j} d x^{i} d x^{j}$ is a smooth tensor field on $(0, T) \times S^{d-1}$ for some $T>0$, such that the restriction of $\gamma$ to $\partial M$ is a smooth tensor on $S^{d-1}$, i.e., $\left.\gamma\right|_{\partial M}=$ $\gamma(0, y)_{A B} d y^{A} d y^{B}$. The following lemma shows that after a change of conformal gauge we may assume that $\tilde{g}$ is in Gauss coordinates based on $\partial M$. 
Lemma 3.10. Consider the conformally compact metric $g=\sinh ^{-2}(t) \tilde{g}$. There is a conformal gauge change so that $g$ takes the form $g=\sinh ^{-2}(\hat{t}) \hat{\tilde{g}}$, where $\hat{t}(p)=$ $d_{\hat{\tilde{g}}}(p, \partial M)$, and $\hat{\tilde{g}}$ is of the form

$$
\hat{\tilde{g}}=d \hat{t}^{2}+h_{0}+\hat{t}^{d} \hat{\gamma}
$$

where $\hat{\gamma}=\hat{\gamma}(\hat{t}, y)_{A B} d y^{A} d y^{B}$ is a $\hat{t}$-dependent tensor field on $S^{d-1}$, such that $\left.\hat{\gamma}\right|_{\partial M}=\left.\gamma\right|_{\partial M}$. In particular, $g$ is asymptotically hyperbolic in the sense of Definition 3.1, with mass aspect tensor $k=\left.\gamma\right|_{\partial M}$.

Proof. Let $\rho=\sinh (t)$. Arguing as in [2, Section 5], we shall find a function $\theta$ such that $\hat{\rho}:=\theta \rho=\sinh (\hat{t})$, where $\hat{t}=d_{\hat{\tilde{g}}}(p, \partial M)$, is the distance to the boundary in the metric $\hat{\tilde{g}}$. This is equivalent to the condition that $\hat{f}:=\operatorname{arcsinh}(\hat{\rho})=\hat{t}$, with $|d \hat{f}|_{\hat{\tilde{g}}}=1$. A calculation as in the proof of [2, Lemma 5.3] shows that this condition is equivalent to the equation

$$
\rho \tilde{g}(d \theta, d \theta)+2 \theta \tilde{g}(d \theta, d \rho)=\theta^{4} \rho+\theta^{2} a
$$

where $a=\rho^{-1}(1-\tilde{g}(d \rho, d \rho))$.

For $\tilde{g}$ of the form (3.70), we have $a=-\rho+O\left(t^{d}\right)$. Equation (3.71) is a system of first order partial differential equations, with characteristics transversal to $\partial M=\{t=0\}$, and satisfies the conditions for existence of solutions with initial condition $\theta=1$ at $\partial M$, see [25, volume 5, pp. 39-40]. Hence there is a small neighborhood $U$ of $\partial M$, and a solution $\theta$ to (3.71) on $U$.

We shall need the following fact.

Claim. $\theta=1+t^{d+1} w$, where $w$ is smooth up to $\partial M$.

The proof of the claim is straightforward and is left to the reader. Now we have that $\hat{t}=\operatorname{arcsinh}(\theta \sinh (t))=t\left[1+O\left(t^{d+1}\right)\right]$, and hence

$$
t=\hat{t}\left[1+O\left(\hat{t}^{d+1}\right)\right]
$$

where the $O\left(t^{d+1}\right)$ and $O\left(\hat{t}^{d+1}\right)$ terms are smooth functions of $(t, y)$ and $(\hat{t}, y)$, respectively. It is straightforward to verify that

$$
\sinh ^{-2}(t)=\sinh ^{-2}(\hat{t})\left[1+\hat{t}^{d+1}\right]
$$

and $g=\sinh ^{-2}(\hat{t}) \hat{\tilde{g}}$, with

$$
\hat{\tilde{g}}=d \hat{t}^{2}+h_{0}+\hat{t}^{d} \hat{\gamma}
$$

where $\hat{\gamma}$ has the property that $\hat{\gamma}(0, y)=\gamma(0, y)$.

By construction, $\hat{t}$ is the distance to $\partial M$, and hence the above is the form of $\hat{\tilde{g}}$ in Gauss coordinates, based on $\partial M$. It follows that $\hat{\gamma}$ is a $\hat{t}$-dependent tensor on $S^{d-1}$. 
3.2.2. Conformal deformation. Assume $(M, g)$ is asymptotically hyperbolic in the sense of Definition 3.1. Then, in slightly different notation, $(M, g)$ has a conformal compactification $(\tilde{M}, \tilde{g})$ with conformal boundary $\partial \tilde{M}$ the round sphere, and such that near $\partial \tilde{M}, g$ has the form,

$$
g=\rho^{-2} \tilde{g}
$$

where $\rho=\sinh (t)$ and

$$
\tilde{g}=d t^{2}+h_{0}+t^{d} \gamma
$$

where $h_{0}$ is the standard metric on $S^{d-1}$ and $\gamma=\gamma(t, \cdot)$ is a $t$-dependent family of metrics on $S^{d-1}$ smooth up to $\partial \tilde{M}$. Note that the mass aspect tensor is given by, $k=\left.\gamma_{A B}\right|_{\partial \tilde{M}}$.

Let $h=h_{0}+t^{d} \gamma$ be the metric induced on the level sets of $t$, and let $\mathrm{s}$ denote the scalar curvature defined with respect to $h$. Further, let $K_{i j}=\frac{1}{2} \partial_{t} h_{i j}$. The only nonvanishing components of $K$ are $K_{A B}=\frac{1}{2} d t^{d-1} \gamma_{A B}+O\left(t^{d}\right)$.

Let $\widetilde{\nabla}$ denote the covariant derivative defined with respect to $\tilde{g}$ and let $\tilde{S}$ denote the scalar curvature of $\tilde{g}$. The formula for the scalar curvature of conformally related metrics gives,

$$
S=-d(d-1) \widetilde{\nabla}_{l} \rho \widetilde{\nabla}^{l} \rho+(2 d-2) \rho \widetilde{\nabla}^{l} \widetilde{\nabla}_{l} \rho+\rho^{2} \tilde{S} .
$$

Claim. S has the asymptotic form,

$$
S=-d(d-1)+O\left(t^{d+1}\right) .
$$

Indeed, by Taylor's theorem, we have $\mathrm{s}=S\left[h_{0}\right]+O\left(t^{d}\right)$, and hence,

$$
\begin{aligned}
\tilde{S} & =\mathrm{s}-2 h_{0}^{A B} \partial_{t} K_{A B}-\left(h_{0}^{A B} K_{A B}\right)^{2}+3 K_{A B} K^{A B} \\
& =(d-1)(d-2)-d(d-1) t^{d-2} h_{0}^{A B} \gamma_{A B}+O\left(t^{d-1}\right) .
\end{aligned}
$$

Further, using $\rho=\sinh t$,

$$
\begin{aligned}
\widetilde{\nabla}^{l} \widetilde{\nabla}_{l} \rho & =\sinh (t)-\tilde{g}^{i j} \widetilde{\Gamma}_{i j}^{t} \cosh (t) \\
& =\sinh (t)+h_{0}^{A B} K_{A B}+O\left(t^{d}\right) \\
& =\sinh (t)+\frac{d}{2} t^{d-1} h_{0}^{A B} \gamma_{A B}+O\left(t^{d}\right) .
\end{aligned}
$$

Finally, we note that $\widetilde{\nabla}^{l} \rho \widetilde{\nabla}_{l} \rho=\cosh ^{2}(t)$. Putting this together, one finds after a few manipulations that the terms involving the mass aspect function, $\mu=$ $h_{0}^{A B}\left(\left.\gamma_{A B}\right|_{\partial \tilde{M}}\right)$, in $S[g]$, at order $t^{d}$, cancel. Equation 3.74 follows.

By standard results [3, Theorem 1.2], there is a unique positive solution $u$ such that $\lim _{x \rightarrow \infty} u(x)=1$, to the Yamabe equation for prescribed scalar curvature $-d(d-1)$ in dimension $d$,

$$
-\frac{4(d-1)}{d-2} \Delta u+S u+d(d-1) u^{\frac{d+2}{d-2}}=0 .
$$

Let $v=u-1$ and let

$$
\hat{S}=\frac{d-2}{4(d-1)}(S+d(d-1))
$$


Then the Yamabe equation takes the form

$$
-\Delta v+d v+\hat{S} v=-\hat{S}-\mathcal{F}(v)
$$

where

$$
\mathcal{F}(v)=\frac{d(d-2)}{4}\left[(1+v)^{\frac{d+2}{d-2}}-1-\frac{d+2}{d-2} v\right] .
$$

In particular $\mathcal{F}(v)=O\left(v^{2}\right)$. A straightforward application of the maximum principle shows that since $S[g] \geq-d(d-1)$, we have $v \leq 0$ and hence

$$
u \leq 1 \text {. }
$$

Linearizing the Yamabe equation around $u=1$, we obtain the equation

$$
-\Delta \bar{u}+d \bar{u}+\hat{S} \bar{u}=0 .
$$

The indicial exponents of this equation are $-1, d$. It follows that the solution to the Yamabe equation is of the form $u=1+v$ with $v=v_{d, 1} t^{d} \log t+v_{d} t^{d}+$ higher order. However since by Equation (3.74), $\hat{S}=O\left(t^{d+1}\right)$, it follows [1] that $v_{d, 1}=0$, and in fact $v$ is smooth up to boundary, with

$$
v=v_{d} t^{d}+\text { higher order . }
$$

Let $L=-\Delta+d$. Equation (3.76) takes the form

$$
L v=f
$$

with $f$ given by

$$
f=-\hat{S} u-\mathcal{F}(u-1) .
$$

In particular, $f \leq 0$ and $f \neq 0$ except when $\hat{S}=0$. Let $L_{t}$ be the operator defined by

We have

$$
L_{t} u=-\sinh ^{2}(t) \partial_{t}^{2} u+(d-2) \sinh (t) \cosh (t) \partial_{t} u+d u .
$$

$$
L u=L_{t} u-\sinh ^{2}(t) \partial_{t} \sqrt{\operatorname{det} h} \partial_{t} u-\sinh ^{2}(t) \Delta_{h} u,
$$

where $\Delta_{h}$ is the Laplacian on $S^{d-1}$ with respect to the metric $h(t, \cdot)=h_{0}+t^{d} \gamma$. In particular $\Delta_{h}$ involves only $y^{A}$-derivatives.

We now introduce a function $w$ which will be used as a supersolution, in order to control the leading order term in $v$. Let $w=-t^{d}(1+d t)$.

Lemma 3.11. There exists constants $t_{1}=t_{1}(d)>0, A=A(d)>0$ such that

$$
L_{t} w>A t^{d+1}, \text { for } 0<t<t_{*} .
$$

Proof. Using $w=-t^{d}(1+d t)$, we obtain,

$$
\begin{aligned}
L_{t}(w)= & \sinh ^{2}(t)\left[d(d-1) t^{d-2}+d^{2}(d+1) t^{d-1}\right] \\
& -(d-2) \sinh (t) \cosh (t)\left[d t^{d-1}+d(d+1) t^{d}\right]-d\left(t^{d}+d t^{d+1}\right) \\
= & {\left[d(d-1) t^{d}+d^{2}(d+1) t^{d+1}\right]-(d-2)\left[d t^{d}+d(d+1) t^{d+1}\right] } \\
& -\left[d t^{d}+d^{2} t^{d+1}\right]+O\left(t^{d+2}\right) \\
= & d(d+2) t^{d+1}+O\left(t^{d+2}\right),
\end{aligned}
$$

and the lemma follows. 
We have,

$$
L w=L_{t} w-\sinh ^{2}(t) \partial_{t} \sqrt{\operatorname{det} h} \partial_{t} w .
$$

For metrics of the form we are considering, $\operatorname{tr}_{h} \partial_{t} h=O\left(t^{d-1}\right)$. Hence by Lemma 3.11, we have

$$
L w>A t^{d+1}-C t^{2 d}
$$

where $C=C(d, \gamma)$. This means there exists $t_{2}>0, t_{2}=t_{2}(d, \gamma)$ such that

$$
L w>0, \text { for } 0<t<t_{2} .
$$

Lemma 3.12. Let $(M, g)$ be asymptotically hyperbolic, so that (3.72) and (3.73) hold. Let $f$ be a function on $(M, g)$, and assume that $f$ is smooth up to $\partial \tilde{M}$, with fall off $f=O\left(t^{d+1}\right)$. Let $L=-\Delta+d$, and let $v$ be the unique solution to

$$
L v=f
$$

with $v=O\left(t^{d}\right)$. Then $v=v_{d} t^{d}+t^{d+1} J$ with $v_{d}=v_{d}(y)$ smooth on $\partial \tilde{M}$ and $J$ smooth up to $\partial \tilde{M}$. If $f \leq 0, f \neq 0$, then $v_{d}<0$.

Proof. Let $\bar{v}_{a}=a w$ and let $v$ be as in (3.77). We have $f \leq 0$, and hence by the strong maximum principle $v<0$ in the interior of $\tilde{M}$. It follows that there is $\epsilon>0$, $t_{2}>t_{3}>0$, so that

$$
\sup _{y \in S^{d-1}} v\left(t_{3}, y\right)<-\epsilon .
$$

For each $a \geq 0, \bar{v}_{a}$ is a supersolution to $L$ in the region $0<t<t_{3}$ and for $0 \leq a \leq a_{*}$, we have that $\bar{v}_{a}\left(t_{3}\right)>v\left(t_{3}, y\right)$ for $y \in S^{d-1}$. Further, we clearly have $\bar{v}_{a}(0)=v(0, y)=0$ for $y \in S^{d-1}$. It follows from the maximum principle that for small $a, \bar{v}_{a}>v$ in the region $0<t<t_{3}$. Fix an $a$ with this property. Since $\bar{v}_{a}=-a t^{d}+O\left(t^{d+1}\right)$, dividing the inequality, $v \leq \bar{v}_{a}$, by $t^{d}$ and letting $t \searrow 0$ gives $v_{d} \leq-a$.

We are now ready to state the following analogue of a well-known result in the asymptotically flat setting (cf., [20]).

Proposition 3.13. Let $(M, g)$ be asymptotically hyperbolic in the sense of Definition 3.1 , with scalar curvature $S[g] \geq-d(d-1)$, and with strict inequality somewhere. Then there exists a conformally related metric $\hat{g}$ such that

1. $(M, \hat{g})$ is asymptotically hyperbolic,

2. $S[\hat{g}]=-d(d-1)$, and

3. $\mu[\hat{g}]<\mu[g]$,

where $\mu[g], \mu[\hat{g}]$ are the mass aspect functions of $(M, g),(M, \hat{g})$, respectively.

Proof. Lemma 3.12, together with the discussion prior to Lemma 3.11, shows that the solution $u$ to the Yamabe equation is of the form

$$
u=1+u_{d} t^{d}+t^{d+1} J
$$


with $u_{d}=u_{d}(y)<0$, and with $J$ smooth up to $\partial \tilde{M}$. Then, after a change of coordinates, $\hat{g}=u^{4 /(d-2)} g$ can be brought into the form,

$$
\hat{g}=\sinh ^{-2}(t)\left(d t^{2}+h_{0}+t^{d}\left(\gamma+\frac{4}{d-2}\left(1+\frac{1}{d}\right) u_{d} h_{0}\right)+t^{d+1} z\right)
$$

where $z=z_{i j} d x^{i} d x^{j}$ is smooth up to $\partial \tilde{M}$.

By Lemma 3.10, after a change of conformal gauge, we have

$$
\hat{g}=\sinh ^{-2}(\hat{t})\left(d \hat{t}^{2}+h_{0}+\hat{t}^{d}\left(\hat{\gamma}+\frac{4}{d-2}\left(1+\frac{1}{d}\right) u_{d} h_{0}\right)\right)
$$

where $\hat{\gamma}=\hat{\gamma}(\hat{t}, y)_{A B} d y^{A} d y^{B}$ is smooth up to $\partial \tilde{M}$ and $\hat{\gamma}(0, y)=\gamma(0, y)$. It follows from the above that the mass aspect functions satisfy,

$$
\mu[\hat{g}]=\mu[g]+\frac{4(d-1)}{d-2}\left(1+\frac{1}{d}\right) u_{d}<\mu[g],
$$

since $u_{d}<0$.

For the purpose of establishing Theorem 3.9, we need the following immediate consequence of Propositions 3.3 and 3.13 .

Corollary 3.14. Let $(M, g)$ be as in Theorem 3.9 ; in particular, assume $\mu[g]=0$. Then $g$ has constant scalar curvature $S[g]=-d(d-1)$.

Proof. Suppose $S[g]>-d(d-1)$ somewhere. Then, by Proposition 3.13, there exists a conformally related metric $\hat{g}$ such that $(M, \hat{g})$ is asymptotically hyperbolic, $S[\hat{g}]=-d(d-1)$, and $\mu[\hat{g}]<\mu[g]=0$. But this directly contradicts Proposition 3.3 .

3.2.3. Deforming the metric. Now we will show that if $g$ has constant scalar curvature $S=-d(d-1)$ and vanishing mass aspect function, then it is Einstein, $\operatorname{Ric}_{g}=-(d-1) g$. Thus, let $(M, g)$ be as in Theorem 3.9, and assume $S[g]=-d(d-1)$.

Let

$$
\widehat{\operatorname{Ric}}=\operatorname{Ric}-\frac{S}{d} g
$$

denote the traceless part of Ric. Note that since $g$ has constant scalar curvature Ric and $\widehat{\mathrm{Ric}}$ have vanishing divergence.

For the subsequent analysis, we shall need detailed information about the asymptotic behavior of $\widehat{\mathrm{Ric}}$.

Lemma 3.15. Let $(M, g)$ be as in Theorem 3.9 (so that (3.72) and (3.73) hold, and the mass aspect vanishes). Then

$$
\widehat{\operatorname{Ric}}=-\frac{d}{2} t^{d-2} \gamma+t^{d-1} z
$$

where $z=z_{i j} d x^{i} d x^{j}$. 
Proof. Let $\widetilde{\text { Ric }}, \widetilde{\nabla}$ denote the Ricci tensor and covariant derivative defined with respect to $\tilde{g}$. The conformal transformation formula for Ricci curvature is

$$
\operatorname{Ric}_{i j}=\widetilde{\operatorname{Ric}}_{i j}+\rho^{-1}\left[(d-2) \widetilde{\nabla}_{i} \widetilde{\nabla}_{j} \rho+\widetilde{\nabla}^{l} \widetilde{\nabla}_{l} \rho \tilde{g}_{i j}\right]-(d-1) \rho^{-2} \widetilde{\nabla}_{l} \rho \widetilde{\nabla}^{l} \rho \tilde{g}_{i j}
$$

where in the right hand side, indices are raised with $\tilde{g}$.

Note that if $\gamma=0$, then $\widetilde{\operatorname{Ric}}=(d-2) h_{0}$ and the only nonvanishing terms in the formula for Ric are

$$
\begin{aligned}
\operatorname{Ric}_{t t} & =(d-1) \sinh ^{-2}(t) \\
\operatorname{Ric}_{A B} & =(d-1) \sinh ^{-2}(t) h_{0 A B} .
\end{aligned}
$$

Now we consider the case with nonvanishing $\gamma$, but with vanishing mass aspect function, i.e., $\mu=\left.h_{0}^{A B} \gamma_{A B}\right|_{t=0}=0$.

Let $K_{i j}=\frac{1}{2} \partial_{t} \tilde{g}_{i j}$. Then the nonvanishing terms in $K$ are $K_{A B}=\frac{1}{2} d t^{d-1} \gamma_{A B}+$ $O\left(t^{d}\right)$. Let $h, \not \nabla$, ric denote the induced metric, covariant derivative and Ricci tensor on the level sets $M_{t}$ of $t$. We use coordinates $y^{A}$ on these level sets, and raise and lower indices with $h$. Note that $h=h_{0}+t^{d} \gamma$, and hence, since ric involves no $t$-derivatives, we have by Taylor's theorem,

$$
\text { ric }=\operatorname{Ric}\left[h_{0}\right]+O\left(t^{d}\right)=(d-2) h_{0}+O\left(t^{d}\right) .
$$

We have from the Gauss, Codazzi, and second variation equations

$$
\begin{aligned}
\widetilde{\operatorname{Ric}}_{t t} & =-h^{A B} \partial_{t} K_{A B}+K_{A C} K^{C}{ }_{B} \\
& =-\frac{1}{2} d(d-1) t^{d-2} h^{A B} \gamma_{A B}+O\left(t^{d-1}\right)
\end{aligned}
$$

which using $\mu=0$ gives,

$$
\begin{aligned}
& =O\left(t^{d-1}\right), \\
\widetilde{\operatorname{Ric}}_{t A} & =\nabla^{B} K_{B A}-\nabla_{A}\left(h^{B C} K_{B C}\right) \\
& =O\left(t^{d}\right), \\
\widetilde{\operatorname{Ric}}_{A B} & =\operatorname{ric}_{A B}-\partial_{t} K_{A B}+2 K_{A C} K^{C}{ }_{B}-K_{A B} h^{C D} K_{C D} \\
& =(d-2) h_{0}-\frac{1}{2} d(d-1) t^{d-2} \gamma_{A B}+O\left(t^{d-1}\right) .
\end{aligned}
$$

Thus we have

$$
\widetilde{\mathrm{Ric}}=(d-2) h_{0}-\frac{1}{2} d(d-1) t^{d-2} \gamma+O\left(t^{d-1}\right) .
$$

We next consider the remaining terms

$$
B_{i j}=\rho^{-1}\left[(d-2) \widetilde{\nabla}_{i} \widetilde{\nabla}_{j} \rho+\widetilde{\nabla}^{l} \widetilde{\nabla}_{l} \rho \tilde{g}_{i j}\right]-(d-1) \rho^{-2} \widetilde{\nabla}_{l} \rho \widetilde{\nabla}^{l} \rho \tilde{g}_{i j} .
$$

Recall that since we are in a Gauss foliation, the only non-vanishing terms in $\widetilde{\Gamma}_{i j}^{t}$ are

$$
\widetilde{\Gamma}_{A B}^{t}=-K_{A B}
$$


We have

$$
\begin{aligned}
B_{t t}= & -(d-1) \sinh ^{-2}(t) \\
B_{t A}= & 0 \\
B_{A B}= & \sinh ^{-1}(t)\left[(d-2) K_{A B} \cosh (t)+\sinh (t) h_{A B}\right] \\
& -(d-1) \sinh ^{-2}(t) \cosh ^{2}(t) h_{A B} \\
= & -(d-1) \sinh ^{-2}(t) h_{0 A B}+\frac{1}{2} d(d-2) t^{d-2} \gamma_{A B}+O\left(t^{d-1}\right) .
\end{aligned}
$$

This shows that

$$
\operatorname{Ric}[g]=-(d-1) g-\frac{d}{2} t^{d-2} \gamma+O\left(t^{d-1}\right)
$$

which gives the Lemma.

Consider the curve,

$$
\lambda_{s}=u_{s}^{4 /(d-2)} g_{s}
$$

where, for $s$ small, $g_{s}$ is the smooth curve of metrics, $g_{s}=g-s \widehat{\operatorname{Ric}}[g]$, and $u_{s}$ is the conformal factor such that $S\left[\lambda_{s}\right]=-d(d-1)$. Note that $u_{0}=1$. By Lemmas 3.10, 3.15 , and our earlier discussion on the asymptotic form of solutions to the Yamabe equation, $\lambda_{s}$ is asymptotically hyperbolic in the sense of Definition 3.1. Let $\mu_{s}$ denote the mass aspect function of $\lambda_{s}$.

Let $\bar{u}=\left.\frac{\partial u_{s}}{\partial s}\right|_{s=0}$. Then, by differentiating the Yamabe equation (3.75), with $u=u_{s}$ and $g=g_{s}$, with respect to the parameter $s$, we obtain the equation,

$$
-\Delta \bar{u}+d \bar{u}=-|\widehat{\operatorname{Ric}}|^{2} \text {. }
$$

By Lemma 3.12, we have

$$
\bar{u}=\bar{u}_{d} t^{d}+O\left(t^{d+1}\right),
$$

with $\bar{u}_{d}=\bar{u}_{d}(y)<0$ if $\widehat{\operatorname{Ric}} \neq 0$.

\section{Lemma 3.16.}

$$
\left.\partial_{s} \mu_{s}\right|_{s=0}=\frac{4(d-1)}{d-2}\left(1+\frac{1}{d}\right) \bar{u}_{d} .
$$

Proof. Clearly, $\alpha=\left.\partial_{s} \mu_{s}\right|_{s=0}$ is of the form $\alpha=\alpha_{\widehat{\mathrm{Ric}}}+\alpha_{u}$, where

$$
\alpha_{\widehat{\mathrm{Ric}}}=\left.\partial_{s} \mu\left(g_{s}\right)\right|_{s=0}
$$

and

$$
\alpha_{u}=\left.\partial_{s} \mu\left(u_{s}^{4 /(d-2)} g\right)\right|_{s=0} .
$$

Let $g_{s}=\sinh ^{-2}(t) \tilde{g}_{s}$. In order to determine the $s$-dependence of the mass aspect function we consider $\tilde{g}_{s}$. It follows from Lemma 3.15 that

$$
\sinh ^{2}(t) \partial_{s} \tilde{g}_{s}=\frac{d}{2} t^{d} \gamma+O\left(t^{d+1}\right)
$$

Since by assumption $\left.\operatorname{tr}_{h_{0}} \gamma\right|_{\partial M}=0$, we have $\alpha_{\widehat{\mathrm{Ric}}}=0$. It follows that the first order change in the mass aspect function of $\lambda_{s}$ is given by $\alpha_{u}$, which clearly is determined by the first order change in the conformal factor $u_{s}$. The result follows. 
We are now ready to complete the proof of Theorem 3.9.

Proof of Theorem 3.9. Let $(M, g)$ be as in Theorem 3.9. Recall $d=n+1$. By Corollary 3.14, $S[g]=-d(d-1)$. Suppose that $g$ is not Einstein, i.e., $\widehat{\operatorname{Ric}} \neq 0$. Let $\lambda_{s}=u_{s}^{4 /(d-2)} g_{s}$ as in (3.80). As previously observed, $\lambda_{s}$ is asymptotically hyperbolic with scalar curvature $S\left[\lambda_{s}\right]=-d(d-1)$, and with mass aspect $\mu_{s}$. By Lemma 3.16, $\left.\partial_{s} \mu_{s}\right|_{s=0}<0$, and hence for small $s>0, \lambda_{s}$ has negative mass aspect function. But in view of Proposition 3.3, $\mu_{s}<0$ gives a contradiction, and hence it must hold that $\widehat{\mathrm{Ric}}=0$. We can now apply the rigidity result of Qing [21] (see also $[4,7])$ to conclude that in fact $(M, g)$ is isometric to hyperbolic space. This concludes the proof of the positive mass theorem in the case of vanishing mass aspect function.

Naturally, it would be desirable to find a way to remove the sign condition on the mass aspect from our positive mass result. Within the context of the approach taken in this paper, one possible way to accomplish this would be to extend the results of Corvino-Schoen [11,12] and Chruściel-Delay [9] on initial data deformations to the asymptotically hyperbolic setting. Starting from Proposition 3.13, the aim would be to deform the time-symmetric initial data to be exactly Schwarzschild-AdS outside a compact set, without changing the scalar curvature and the sign of the mass. Starting from Schwarzschild-AdS with mass $m<0$, the deformation result of Section 3.1 is then easily proved.

\section{Acknowledgements}

We thank Piotr Chruściel for comments and discussion. This work was initiated at the Conference on Mathematical Aspects of Gravitation at the Mathematical Research Institute in Oberwolfach in 2003. We thank the Institute for its hospitality and support. This work was supported in part by NSF grants DMS-0407732 and DMS-0405906.

\section{References}

[1] L. Andersson and P. T. Chruściel, Solutions of the constraint equations in general relativity satisfying "hyperboloidal boundary conditions", Dissertationes Math. (Rozprawy Mat.) 355 (1996), 100.

[2] L. Andersson and M. Dahl, Scalar curvature rigidity for asymptotically locally hyperbolic manifolds, Ann. Global Anal. Geom. 16 (1998), no. 1, 1-27.

[3] L. Andersson, P. T. Chruściel and H. Friedrich, On the regularity of solutions to the Yamabe equation and the existence of smooth hyperboloidal initial data for Einstein's field equations, Commun. Math. Phys. 149 (1992), 587-612.

[4] V. Bonini, P. Miao, and J. Qing, Ricci curvature rigidity for weakly asymptotically hyperbolic manifolds, Comm. Anal. Geom. 14 (2006), no. 3, 603-612. 
[5] M. Cai, Volume minimizing hypersurfaces in manifolds of nonnegative scalar curvature, Minimal surfaces, geometric analysis and symplectic geometry (Baltimore, MD, 1999), Adv. Stud. Pure Math., vol. 34, Math. Soc. Japan, Tokyo, 2002, pp. 1-7.

[6] M. Cai and G. J. Galloway, Rigidity of area minimizing tori in 3-manifolds of nonnegative scalar curvature, Comm. Anal. Geom. 8 (2000), no. 3, 565-573.

[7] M. Cai and J. Qing, On the rigidity of AdS spacetime, preprint (2006).

[8] U. Christ and J. Lohkamp, Singular minimal hypersurfaces and scalar curvature, math.DG/0609338 (2006).

[9] P. T. Chruściel and E. Delay, On mapping properties of the general relativistic constraints operator in weighted function spaces, with applications, Mém. Soc. Math. Fr. (N.S.) (2003), no. 94, vi+103.

[10] P. T. Chruściel and M. Herzlich, The mass of asymptotically hyperbolic Riemannian manifolds, Pacific J. Math. 212 (2003), no. 2, 231-264.

[11] J. Corvino, Scalar curvature deformation and a gluing construction for the Einstein constraint equations, Comm. Math. Phys. 214 (2000), no. 1, 137-189.

[12] J. Corvino and R. M. Schoen, On the asymptotics for the vacuum Einstein constraint equations, J. Differential Geom. 73 (2006), no. 2, 185-217.

[13] E. Delay, Analyse précisée d'équations semi-linéaires elliptiques sur l'espace hyperbolique et application à la courbure scalaire conforme, Bull. Soc. Math. France 125 (1997), no. 3, 345-381.

[14] H. Federer, Geometric measure theory, Die Grundlehren der mathematischen Wissenschaften, Band 153, Springer-Verlag New York Inc., New York, 1969.

[15] G. W. Gibbons, S. W. Hawking, G. T. Horowitz, and M. J. Perry, Positive mass theorems for black holes, Comm. Math. Phys. 88 (1983), no. 3, 295-308.

[16] J. L. Kazdan and F. W. Warner, Existence and conformal deformation of metrics with prescribed Gaussian and scalar curvatures, Ann. of Math. (2) 101 (1975), 317-331.

[17] J. Lohkamp, The higher dimensional positive mass theorem I, math.DG/0608795 (2006).

[18] J. Lohkamp, Scalar curvature and hammocks, Math. Ann. 313 (1999), no. 3, 385-407.

[19] M. Min-Oo, Scalar curvature rigidity of asymptotically hyperbolic spin manifolds, Math. Ann. 285 (1989), no. 4, 527-539.

[20] N. Ó Murchadha and J. W. York Jr., Gravitational energy, Phys. Rev. D 10 (1974), no. 8, 2345-2357.

[21] J. Qing, On the rigidity for conformally compact Einstein manifolds, Int. Math. Res. Not. (2003), no. 21, 1141-1153.

[22] R. Schoen and S. T. Yau, On the structure of manifolds with positive scalar curvature, Manuscripta Math. 28 (1979), no. 1-3, 159-183.

[23] R. Schoen and S. T. Yau, On the proof of the positive mass conjecture in general relativity, Comm. Math. Phys. 65 (1979), no. 1, 45-76.

[24] Y. Shi and G. Tian, Rigidity of asymptotically hyperbolic manifolds, Comm. Math. Phys. 259 (2005), no. 3, 545-559.

[25] M. Spivak, A comprehensive introduction to differential geometry, second ed., Publish or Perish Inc., Wilmington, Del., 1979. 
[26] X. Wang, The mass of asymptotically hyperbolic manifolds, J. Differential Geom. 57 (2001), no. 2, 273-299.

[27] E. Witten and S.-T. Yau, Connectedness of the boundary in the AdS/CFT correspondence, Adv. Theor. Math. Phys. 3 (1999), no. 6, 1635-1655 (2000).

[28] S. T. Yau, Geometry of three manifolds and existence of black hole due to boundary effect, Adv. Theor. Math. Phys. 5 (2001), no. 4, 755-767.

Lars Andersson*, Mingliang Cai, and Gregory J. Galloway

Department of Mathematics

University of Miami

Coral Gables, FL 33124

USA

and

* Max-Planck-Institut für Gravitationsphysik

Am Mühlenberg 1

D-14476 Postdam

Germany

e-mail: larsa@math.miami.edu

m. cai@math.miami.edu

galloway@math.miami .edu

Communicated by Sergiu Klainerman.

Submitted: March 16, 2007.

Accepted: June 14, 2007. 